\title{
Rotating Metal Foam Structures for Performance Enhancement of Double-pipe Heat Exchangers
}

DOI:

10.1016/j.ijheatmasstransfer.2016.09.055

\section{Document Version}

Accepted author manuscript

Link to publication record in Manchester Research Explorer

\section{Citation for published version (APA):}

Alhusseny, A., Turan, A., \& Nasser, A. (2017). Rotating Metal Foam Structures for Performance Enhancement of Double-pipe Heat Exchangers. International Journal of Heat and Mass Transfer.

https://doi.org/10.1016/j.ijheatmasstransfer.2016.09.055

\section{Published in:}

International Journal of Heat and Mass Transfer

\section{Citing this paper}

Please note that where the full-text provided on Manchester Research Explorer is the Author Accepted Manuscript or Proof version this may differ from the final Published version. If citing, it is advised that you check and use the publisher's definitive version.

\section{General rights}

Copyright and moral rights for the publications made accessible in the Research Explorer are retained by the authors and/or other copyright owners and it is a condition of accessing publications that users recognise and abide by the legal requirements associated with these rights.

\section{Takedown policy}

If you believe that this document breaches copyright please refer to the University of Manchester's Takedown Procedures [http://man.ac.uk/04Y6Bo] or contact uml.scholarlycommunications@manchester.ac.uk providing relevant details, so we can investigate your claim.

\section{OPEN ACCESS}




\title{
Rotating Metal Foam Structures for Performance Enhancement of Double-pipe Heat Exchangers
}

\author{
Ahmed Alhusseny ${ }^{1,2, *}$, Ali Turan ${ }^{1}$, and Adel Nasser ${ }^{1}$ \\ ${ }^{1}$ School of Mechanical, Aerospace and Civil Engineering, The University of Manchester, Manchester, UK \\ ${ }^{2}$ Mechanical Engineering Department, College of Engineering, University of Kufa, Najaf, Iraq
}

\begin{abstract}
In order to enhance the amount of heat transported in a double-pipe heat exchanger, a compound enhancement is proposed incorporating both active and passive methods. The first one is through introducing secondary flows in the vicinity of the conducting surface using metal foam guiding vanes, which are fixed obliquely and rotating coaxially to trap fluid particles while rotation and then force them to flow over the conducting surface. The other is via covering the conducting surface between the two pipes with a metal foam layer to improve the heat conductance across it. This proposal is examined numerically by studying the three-dimensional, steady, incompressible, and laminar convective fluid flow in a counter-flow double-pipe heat exchanger partially filled with high porosity metal foam and rotating coaxially. With regards to the influence of rotation, both the centrifugal buoyancy and Coriolis forces are considered in the current study. The generalised model is used to mathematically simulate the momentum equations in the porous regions. Moreover, thermal dispersion has been taken into account while considering that fluid and solid phases are in a local thermal non-equilibrium. Computations are performed for a wide range of design parameters influencing the performance achieved such as the operating conditions, the configuration of the guiding vanes utilized, and the geometrical and thermal characteristics of the metal foam utilised. The results are presented by means of the heat exchanger effectiveness, pressure drop, and the overall system performance. The current proposed design has effectively proved its potential to enhance the heat transported considerably in view of the significant savings in the pumping power required compared to the heat exchangers fully filled with metal foams. Furthermore, the data obtained reveal an obvious impact of the design parameters inspected on both the heat exchanged and the pressure loss; and hence, the overall performance obtained. Although the heat exchanger effectiveness can be improved considerably by manipulating the design factors, care must be taken to avoid unnecessary expenses resulted from potential increases in pressure drop.
\end{abstract}

KEYWORDS: Heat Exchanger, Compound Enhancement, Metal Foams, Rotation, Overall Performance

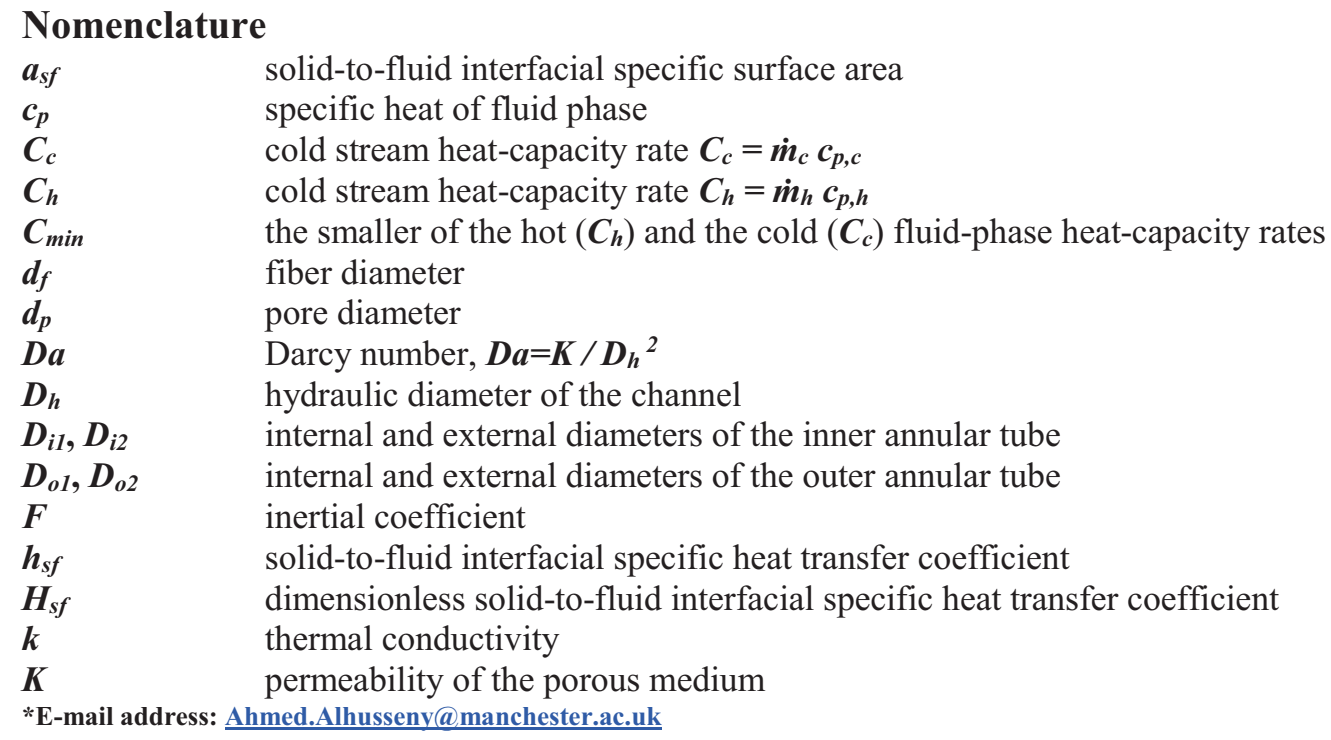




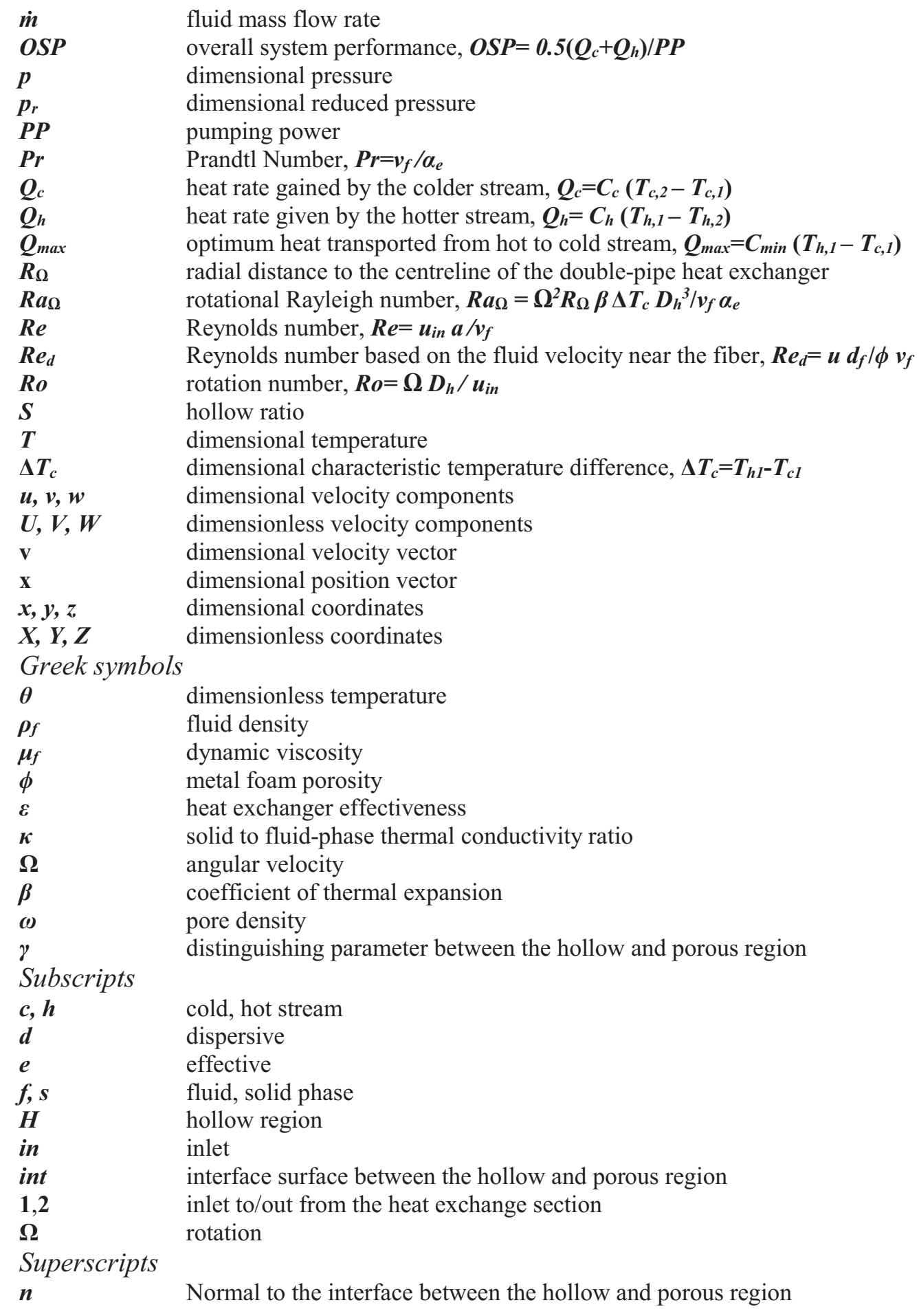

\section{Introduction}

Improving the performance of power generation plants while minimising the environmental damage caused by the excessive use of fossil fuels has become a crucial concern recently. To achieve a high thermal efficiency in power generation systems, heat recirculation is applied between the cold intake and hot exhausts to recover a part of its thermal energy instead of releasing it directly to the environment (Alhusseny and Turan [1]). Heat recirculation is usually accomplished by means of two options. Either a 
recuperative or regenerative heat exchanger is used, depending respectively on whether the heat exchange takes place directly via a thermally conductive surface separating the two streams or through an intermediate storage medium exposed to them alternately (Hewitt et al. [2]).

Enhancing the heat transported in recuperative heat exchangers has acquired increasing attention due to their crucial role in many practical applications found in industry, power plants, and space exploration, as well as many others. Heat transfer enhancement techniques can be classified into active, in which an external power is required; passive, which does not require an external power input; or compound, where two or more of the aforementioned techniques may be employed simultaneously to achieve a larger enhancement than what can be produced using the individual techniques separately (Bergles [3]).

One of the simplest arrangements of recuperators available in industry and practical applications is the double-pipe heat exchanger. It is widely utilised in practical applications, and hence, has been the focus of plenty of studies recently. In the light of economic considerations, many efforts have been made to construct cheaper and smaller but more effective heat exchangers.

Among these investigations, some have been dedicated to renew the boundary layer developing over the heat exchange surface, and hence, enhance the amount of heat transported. For example, it was found that placing propellers inside the inner tube leads to enhance the heat exchanged by up to $250 \%$ and a further enhancement can be achieved through increasing Reynolds number and/or the number of propellers used [4]. Another passive way is to place a strip turbulator twisted in certain angles to touch the inside wall of the inner pipe [5], where heat transfer rate can be improved by $100 \%$ or more through increasing the pitch length. Also, it was found that covering the conducting surface with a porous layer ([6], [7], and [8]) or attaching porous structures to this surface ([9] and [10] can considerably improve the effectiveness achieved.

Convective flows in porous materials have been widely investigated for over the last decades and various aspects were considered for different applications, where their state of art has been summarised extensively by Nield and Bejan [11] as well as Ingham and Pop [12]. However, most studies have been

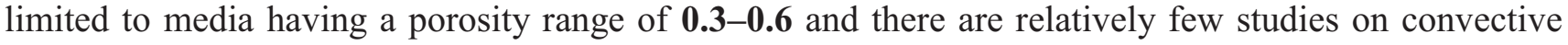
flow phenomena in materials with very high porosity like open-cell metal foams.

High porosity metal foams are usually porous media with low density and novel structural and thermal properties (Tianjian [13]). They offer light weight, high rigidity and strength, and high surface area, which make them able to recycle heat efficiently. Also, their open-cell structure makes them less resistant to the fluids flowing through them, and hence, pressure drop across them is much less than it in the case of flow via packed beds or granular porous media. Therefore and due to their ability to meet the highly thermal demands with no excessive loss in pressure, open-cell metal foams have been utilised in heat exchangers [14], [15] besides internal cooling of both turbine blades [16] and the rotor windings of high-capacity electrical generators [17], [18], and [19]. Thus, it is not surprising to use them recently in double-pipe heat exchangers [20] and [21], where a substantial enhancement in the heat transfer performance has been acquired.

With regards to combined fluid flow and heat transfer in rotating porous media, relevant studies have been motivated by its wide range of practical and fundamental applications in engineering and geophysics. Chemical processing, materials, and food industries, in addition to rotating machinery are just a few examples of its engineering applications (Vadasz [22]). Recently, Alhusseny and Turan [23] presented a numerical study for Coriolis' effect on combined heat and mass transfer in a radially rotating porous channel, where rotation was found to have a negative impact on heat and mass transport. Utilizing metal foams to improve the internal cooling of turbine blades was examined experimentally by Tzeng et al. [24] through filling a radially rotating serpentine channel with aluminum foam. It was found that this proposal enhances the amount of heat transported between the solid and fluid phases, and hence, improves the overall efficiency of cooling process. More recently, Alhusseny and Turan [17] and Alhusseny et al. [18] studied numerically the developing flow through a channel rotating in a parallel- 
mode and fully filled with high porosity metal foam, where effects of rotation, geometrical and thermal characteristics of the fiber used, channel aspect ratio, and thermal dispersion on heat transfer performance were investigated.

Despite the fact that using porous media, which have high thermal conductivity, improves heat transfer considerably, it also results in relatively high pressure losses. Therefore, channels partially filled with permeable materials have been proposed as an alternative to avoid unnecessary pressure drop while keeping the heat transfer at as high rates as possible. For example, and in order to improve the overall enhancement achieved, the effect of utilising rotating cooling channels partially filled with open-cell metal foams was numerically investigated by Alhusseny et al. [19].

The current paper is dedicated to present a proposal to improve the performance of a double-pipe heat exchanger. A compound enhancement method is suggested including both passive, i.e. using metal foam structures, and active technique, by subjecting the system to a coaxial rotation. The benefit gained from using such configuration is checked numerically taking into account the potential losses due to the increase in pressure drop.

\section{Mathematical formulation}

Two steady, incompressible, laminar, and counter-flowing streams are carried in a heat exchanger rotating coaxially and comprised of two annular pipes separated with a $\mathbf{2} \mathbf{m m}$ conducting surface, as shown in Figure 1. The total length of the heat exchange section is $\mathbf{4 0 0} \mathbf{m m}$, while the internal and external diameters of the inner annular tube are $\boldsymbol{D}_{\boldsymbol{i} 1}=\mathbf{2 0}$ and $\boldsymbol{D}_{\boldsymbol{i} \mathbf{2}}=\mathbf{4 0} \mathbf{m m}$, respectively, and their values for the outer pipe are $D_{o 1}=44$ and $D_{o 2}=64 m m$, respectively.

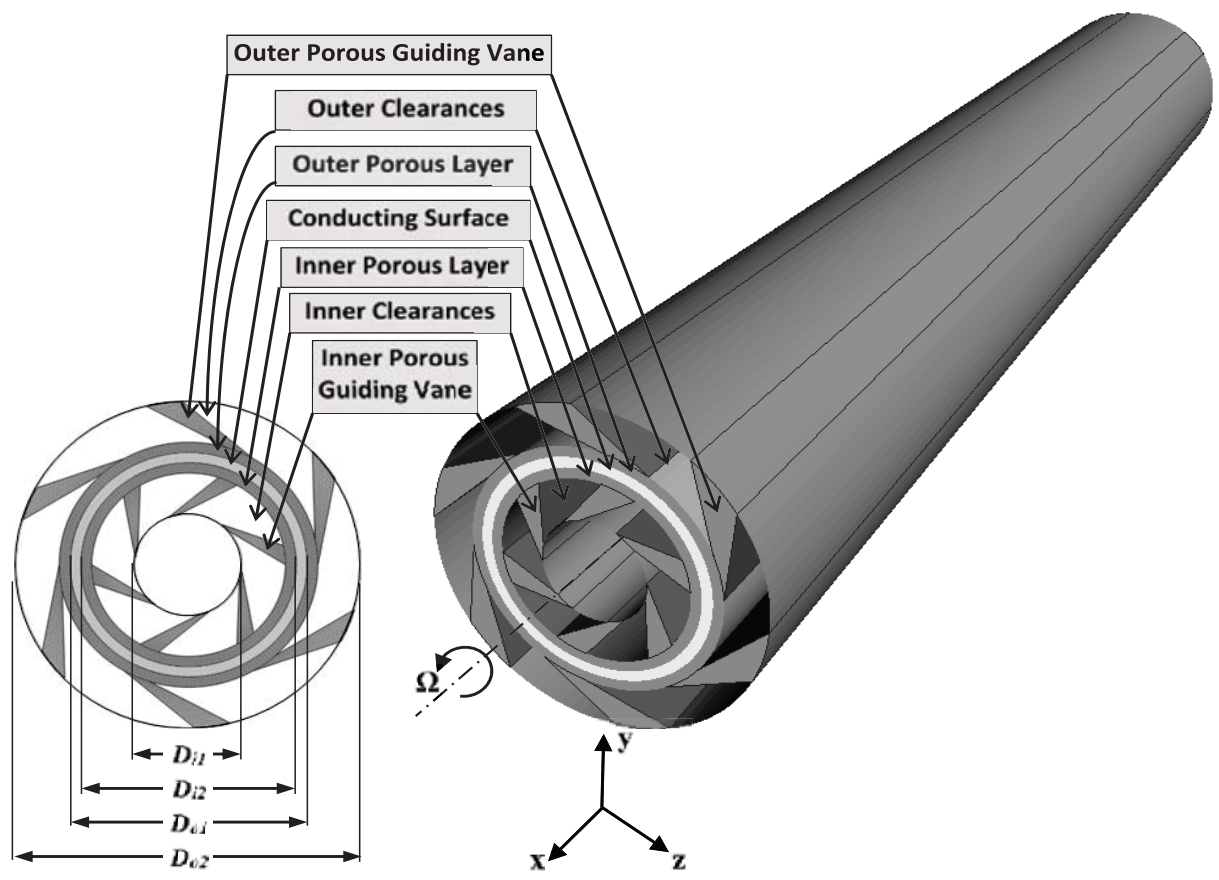

Figure 1- Geometrical shape of the studied problem

The porous material is considered isotropic, homogeneous, and rigid metal foam of high porosity $\phi \geq \mathbf{0 . 8 9}$. The generalised model is employed to simulate the momentum equations considering both the solid and fluid phases to be in local thermal non-equilibrium with each other. In regards to the influence of rotation, both the centrifugal buoyancy and Coriolis forces are taken into account.

Thus, the dimensional forms of conservation equations of mass, momentum, and energy for both fluid and solid-phase as well as the conducting surface are: 


$$
\begin{aligned}
& \begin{array}{l}
\nabla \cdot \mathbf{v}=0 \\
\frac{\rho_{f}}{\phi^{2}}(\mathbf{v} \cdot \nabla) \mathbf{v}= \\
\quad-\nabla p_{r}+\frac{\mu}{\phi} \nabla^{2} \mathbf{v}-\frac{2 \rho_{f} \Omega}{\phi} e_{\Omega} \times \mathbf{v}-\gamma\left(\frac{\mu}{K} \mathbf{v}+\rho_{f} \frac{F}{\sqrt{K}}|\mathbf{v}| \mathbf{v}\right) \\
\quad+\left(\rho_{f}-\rho_{0}\right) \Omega^{2} e_{\Omega} \times\left(e_{\Omega} \times \mathbf{x}\right)
\end{array} \\
& \rho_{f} c_{p}(\mathbf{v} \cdot \nabla) T_{f}=(1-\gamma) k_{f} \nabla^{2} T_{f}+\gamma\left[\left(k_{f e}+k_{d}\right) \nabla^{2} T_{f}+a_{s f} h_{s f}\left(T_{s}-T_{f}\right)\right] \\
& 0=k_{s e} \nabla^{2} T_{s}+a_{s f} h_{s f}\left(T_{f}-T_{s}\right) \\
& 0=k_{w} \nabla^{2} T_{w}
\end{aligned}
$$

In Eqs.[(2), (3)], $\boldsymbol{\gamma}$ is a distinguishing parameter equals to either zero or one depending on whether the flow takes place in the clear or porous regions, respectively; while the medium porosity $\phi$ equals to one just in the clear regions. Also, $\boldsymbol{K}$ and $\boldsymbol{F}$ represent the permeability and inertial coefficient of the metal foam, respectively, where their values are computed according to the model proposed by Calmidi [25] as:

$$
\begin{aligned}
& K=0.00073 d_{p}^{2}(1-\phi)^{-0.224}\left(\frac{d_{f}}{d_{p}}\right)^{-1.11} \\
& F=0.00212(1-\phi)^{-0.132}\left(\frac{d_{f}}{d_{p}}\right)^{-1.63}
\end{aligned}
$$

In addition, $\boldsymbol{k}_{f e}$ and $\boldsymbol{k}_{\boldsymbol{s} e}$ are the effective thermal conductivity of fluid and solid phase, respectively. Their values are computed using the model developed by Boomsma and Poulikakos [26] and then corrected by them [27]. The dispersion conductivity $\boldsymbol{k}_{\boldsymbol{d}}$, which is assumed to be isotropic, is determined based on the model presented by Hunt and Tien [28]:

$k_{d}=C_{d} \rho c_{p} u \sqrt{K}$

Where $\boldsymbol{C}_{\boldsymbol{d}}$ is the coefficient of thermal dispersion and its value was found to be $\mathbf{0 . 0 6}$ as proposed by Calmidi and Mahajan [29]. Finally, $\boldsymbol{a}_{\boldsymbol{s} f}$ and $\boldsymbol{h}_{\boldsymbol{s} f}$ are the solid-fluid interfacial specific surface area and heat transfer coefficient and can be determined using the model suggested by Calmidi and Mahajan [29]. However, the $\boldsymbol{h}_{\boldsymbol{s} f}$ value is estimated using the model proposed by Lu et al. [30] due to its generality in not only relying on the foam porosity and fiber diameter, but being also a function to the pore flow regime as:

$$
N u_{s f}=\frac{h_{s f} d}{k_{f}}=\left\{\begin{array}{l}
0.76 \operatorname{Re}_{d}^{0.4} \operatorname{Pr}^{0.37},\left(1 \leq \operatorname{Re}_{d} \leq 40\right) \\
0.52 \operatorname{Re}_{d}^{0.5} \operatorname{Pr}^{0.37},\left(40 \leq \operatorname{Re}_{d} \leq 10^{3}\right) \\
0.26 \operatorname{Re}_{d}^{0.6} \operatorname{Pr}^{0.37},\left(10^{3} \leq \operatorname{Re}_{d} \leq 2 \times 10^{5}\right)
\end{array}\right\} \text {, where; } d=\left(1-e^{-(1-\phi) / 0.04}\right) d_{f}
$$

Although the governing equations of fluid flow and heat transport either within the metal foams or in the clear zones are not coupled with each other, they are linked together through interface surfaces separating them. So, Eqs.[ (1) - (4)] need to be closed by means of interfacial coupling conditions, where continuity of velocity, shear stress, fluid temperature, and heat flux along the fluid-solid interfaces shown in Figure 2 must be ensured to get meaningful physics ( $\mathrm{Xu}$ et al. [31]). To this end, boundary conditions proposed by Ochoa-Tapia and Whitaker [32] for the fluid-solid interface are used with taking the thermal dispersion into account as detailed in Eqs.(11.a-e) mentioned previously by Alhusseny et al. [19]. 


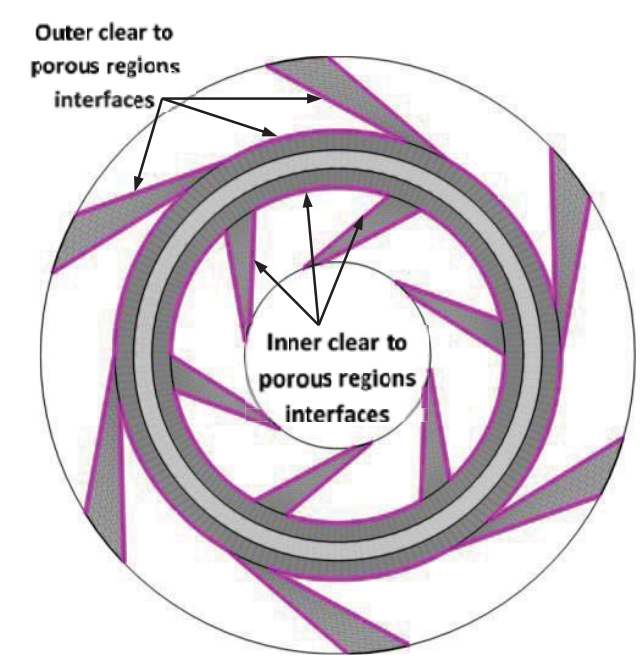

Figure 2- Interfaces between the clear and the porous regions

The boundary conditions employed to solve the aforementioned governing equations are:

$$
\begin{aligned}
& \text { at } x=0 ; \begin{cases}D_{i 1}<D<D_{i 2}: & u=u_{c, i n}, v=w=0, T_{f}=T_{s}=T_{c, i n} \\
D_{i 2}<D<D_{o 1}: & \frac{\partial T_{w}}{\partial x}=0 \\
D_{o 1}<D<D_{o 2}: & \frac{\partial u}{\partial x}=\frac{\partial v}{\partial x}=\frac{\partial w}{\partial x}=\frac{\partial T_{f}}{\partial x}=\frac{\partial T_{s}}{\partial x}=0\end{cases} \\
& \text { at } x=L ; \begin{cases}D_{i 1}<D<D_{i 2}: & \frac{\partial u}{\partial x}=\frac{\partial v}{\partial x}=\frac{\partial w}{\partial x}=\frac{\partial T_{f}}{\partial x}=\frac{\partial T_{s}}{\partial x}=0 \\
D_{i 2}<D<D_{o 1}: & \frac{\partial T_{w}}{\partial x}=0 \\
D_{o 1}<D<D_{o 2}: & u=-u_{h, i n}, v=w=0, T_{f}=T_{s}=T_{h, i n} \\
D=D_{i 1}: \quad u=v=w=0, \frac{\partial T_{f}}{\partial \mathbf{x}_{n}}=\frac{\partial T_{s}}{\partial \mathbf{x}_{n}}=0 \\
D=D_{i 2} ; D_{o 1}: \quad u=v=w=0, k_{s e} \frac{\partial T_{s}}{\partial \mathbf{x}_{n}}+\left(k_{f e}+k_{d}\right) \frac{\partial T_{f}}{\partial \mathbf{x}_{n}}=k_{w} \frac{\partial T_{w}}{\partial \mathbf{x}_{n}} \\
D=D_{o 2}: & u=v=w=0, \frac{\partial T_{f}}{\partial \mathbf{x}_{n}}=\frac{\partial T_{s}}{\partial \mathbf{x}_{n}}=0\end{cases}
\end{aligned}
$$

\section{Solution procedure}

The governing equations are discretised using the finite volume method. The second-order differencing scheme is employed to represent the convection terms in the discretised governing equations, while the problem of pressure-velocity coupling is resolved using SIMPLE algorithm.

A structured grid formed of hexahedral elements was built using Pointwise software, where the stationary clear regions are linked to the rotating clear/porous regions through four interface surfaces as shown in Figure 3. Steep gradients expected at the boundaries including the walls and the interfaces are 
captured by refining the mesh at these regions. Grid dependency was checked by examining four sets of grid including the stationary and rotating regions $(\mathbf{9 1 4 , 6 2 0}, \mathbf{1 , 2 2 2 , 4 2 0}, \mathbf{1 , 5 4 1 , 3 8 0}$, and $\mathbf{1 , 8 3 8 , 0 2 0})$ at $\boldsymbol{R e}_{\text {inner }}=\boldsymbol{R} \boldsymbol{e}_{\text {outer }}=\mathbf{2 0 0 0}, \Omega=500 \mathrm{rpm}, \boldsymbol{k}_{s}=\boldsymbol{k}_{w}, \phi=0.9$, and $\boldsymbol{\omega}=\mathbf{1 0 P P I}$. It is noticed that the deviation in the data obtained becomes marginal between the third and fourth grid set and thereafter. Thus, the mesh size of $(1,541,380)$ can be considered sufficient for the accuracy of the current study.

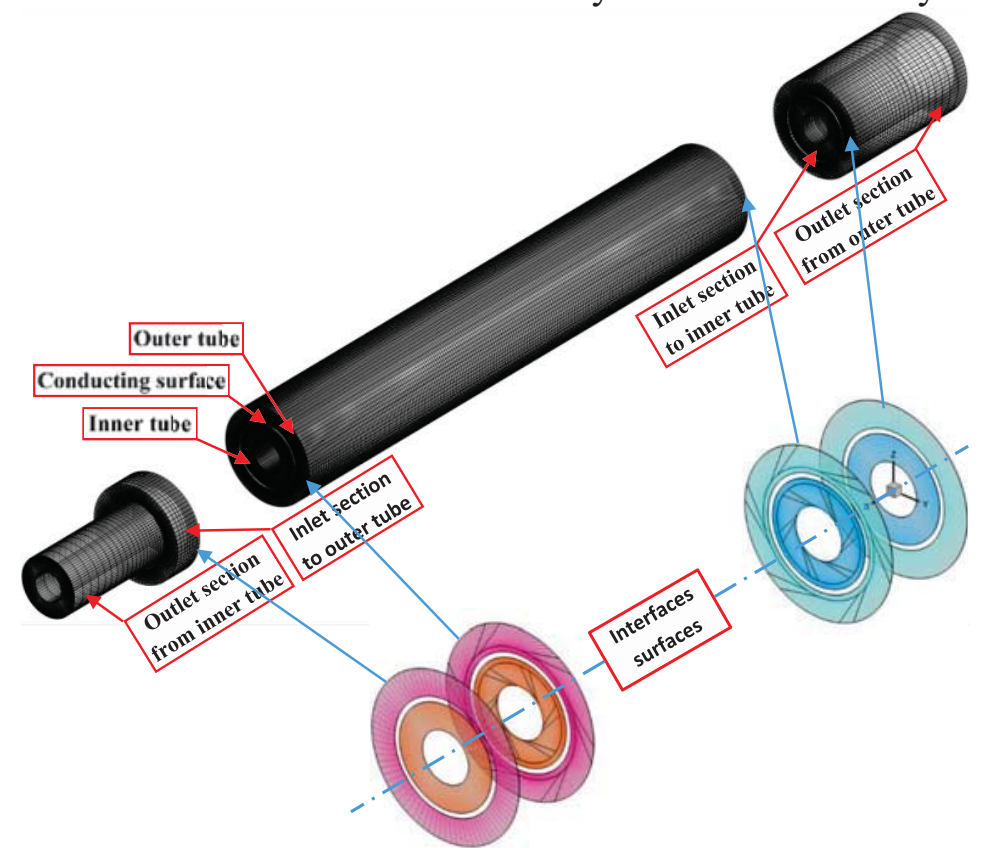

Figure 3- The used grid with the interfaces linking the stationary clear to the rotating porous regions

The "STAR CCM+" commercial CFD code is utilised to simulate the current problem. However, this software does not support using "the local thermal non-equilibrium model" to track the solid-to-fluid interstitial heat transport within the porous regions. To overcome this drawback, the porous region was duplicated into two identical zones representing the solid and fluid phase individually and then heat can be allowed to transfer between the two phases and tracked by creating a heat exchanger interface there. Under-relaxation technique is used in order to avoid divergence during the iterative process, where under-relaxation factors of about (0.3-0.99) are used for the dependent variables. Convergence is measured in terms of the maximum change in the difference between the heat transported from the outer stream to the conducting surface and that transferred from the conducting surface to the inner stream during any iteration as:

$$
\text { Err. }=\frac{Q_{\text {in, Gained }}+Q_{\text {out }, \text { Lost }}}{\left(Q_{\text {in, Gained }}+\operatorname{Abs}\left(Q_{\text {out }, \text { Lost }}\right)\right) / 2}
$$

Where the maximum change allowed for convergence check is $\mathbf{1 0}^{\mathbf{- 5}}$.

Due to the lack of experiments conducted on heat and fluid flow in double-pipe heat exchangers filled with metal foam, the currently computed data were validated with the numerical data presented by $\mathrm{Xu}$ et al. [20] for a stationary heat exchanger. The profiles of dimensionless axial velocity at the exit section of each pipe were compared with the corresponding data from the above mentioned study as shown in Figure 4. Regarding the heat transfer problem, the axial development of the cross-sectional mean temperature for the inner fluid, conducting wall, and the outer fluid was also compared with the corresponding data presented by Xu et al. [20] as shown in Figure 5. Overall, the currently computed results are clearly in excellent agreement with numerical data presented in the above mentioned study. 


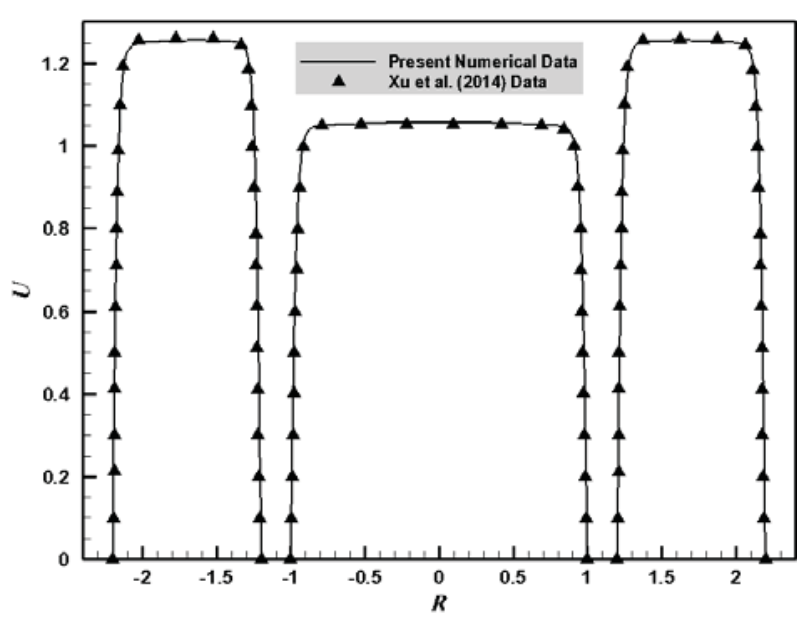

(a) $\omega=5$ PPI

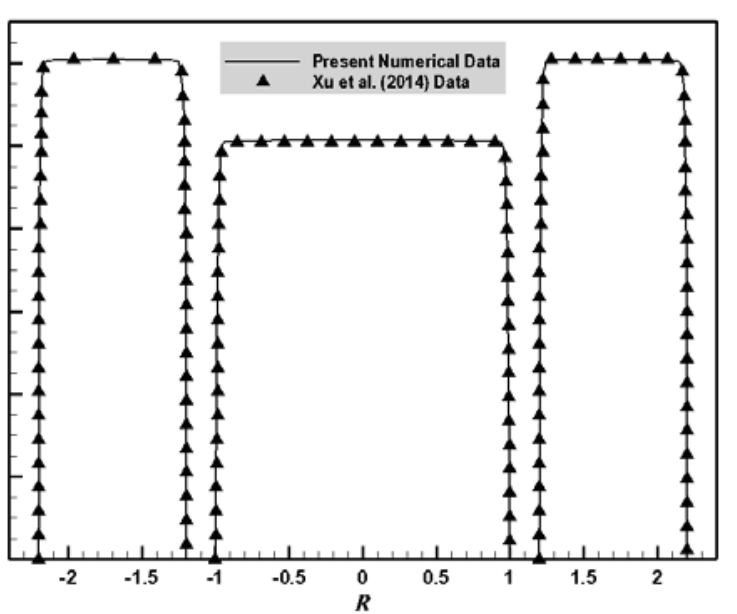

(b) $\omega=30$ PPI

Figure 4- Comparing the dimensionless velocity profile at the exit sections of a double pipe heat exchanger fully filled with metal foam at $\operatorname{Re}_{\text {inner }}=\operatorname{Re}_{\text {outer }}=1000$, and $\phi=0.9$

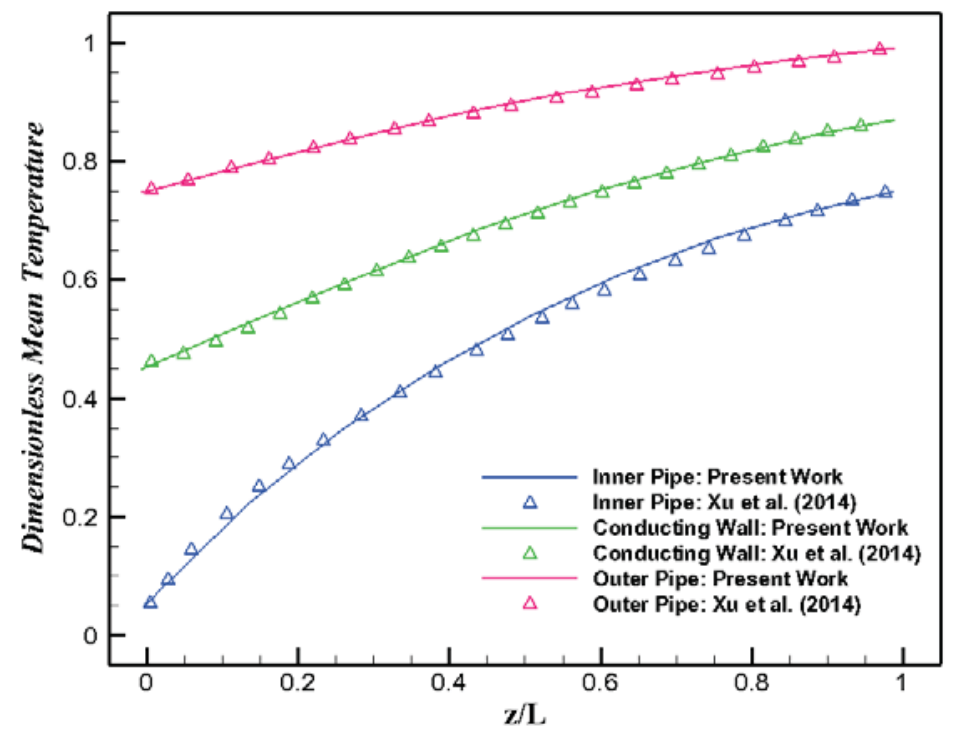

Figure 5- Axial development of the cross-sectional mean temperature for the inner pipe, conducting wall, and the outer pipe of a double pipe heat exchanger fully filled with metal foam at $\operatorname{Re}_{\text {inner }}=\operatorname{Re}_{\text {outer }}=1000, \phi=0.9$, and $\omega=10 P P I$

\section{Results}

The obtained results are presented for a wide range of parameters including the clearance-to-vane size ratio $\boldsymbol{S}$, foam porosity $\phi$, foam thermal conductivity $\boldsymbol{k}_{\boldsymbol{s}}$, Reynolds number ratio $\boldsymbol{R}_{\boldsymbol{e}}$, rotational speed $\boldsymbol{\Omega}$, and characteristic temperature difference $\Delta \boldsymbol{T}_{c}$, while the values of pore density, inner inlet temperature, and outer Reynolds number are kept constant at $\boldsymbol{\omega}=\mathbf{1 0 P P I}, \boldsymbol{T}_{\text {inner }}=\mathbf{3 0} \mathbf{C}^{\circ} \mathrm{C}$, and $\boldsymbol{R e}_{\text {outer }}=\mathbf{2 0 0 0}$, respectively.

The efficiency of heat exchange process is mainly measured in terms of two key design parameters. The first is the effectiveness of the counter-flow heat exchanger, which can be computed as:

$\varepsilon=\frac{Q_{c}}{Q_{\max }}=\frac{Q_{h}}{Q_{\max }}$

Where; 
$Q_{c}=C_{c}\left(T_{c, 2}-T_{c, 1}\right), \quad Q_{h}=C_{h}\left(T_{h, 1}-T_{c, 1}\right), \quad Q_{\max }=C_{\min }\left(T_{h, 1}-T_{c, 1}\right)$

The other key parameter in assessing the overall performance is the total pressure loss $\Delta \boldsymbol{P}_{\boldsymbol{t}}$ occurring across the heat exchanger, which indicates the pumping power required as:

$P P_{t}=P P_{c}+P P_{h}=\dot{V}_{c} \Delta P_{c}+\dot{V}_{h} \Delta P_{h}=\left.\frac{\dot{m}}{\rho}\right|_{c} \Delta P_{c}+\left.\frac{\dot{m}}{\rho}\right|_{h} \Delta P_{h}$

Overall, examining the practical worth of using a specific design or flow characteristics is essential in designing heat exchangers. This can be achieved through introducing a dimensionless performance measure called the overall system performance $\boldsymbol{O S P}$, which is defined as the ratio of the heat exchanged to the pumping power required as:

$O S P=\frac{Q_{\text {Exchanged }}}{P P_{t}}$

This factor can be even used for designing other types of heat exchangers, where as long as the $\boldsymbol{O S P}$ value "or the ratio of the gain to the cost" is higher, the overall performance is better.

\subsection{Influence of rotation on fluid flow and heat transfer development}

Rotation effects on the development of flow field and heat transfer are illustrated in Figure $\mathbf{6}$ for

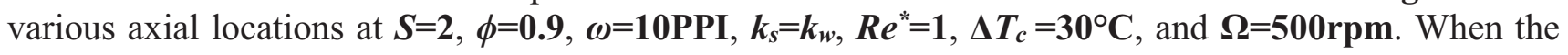
two counter-flowing streams enter to the rotating heat exchange part, the influence of body forces induced by rotation starts to appear, i.e. Coriolis and centrifugal forces, resulting in forming transverse vortices and reorganising the flow patterns in a quite different way from the classical flow behaviour. As a result of Coriolis forces, a main counter-to-rotation vortex is created at the entrance of each of the inner and outer pipe, i.e. $\mathbf{x}=\mathbf{0 . 0 1 L}$ and $\mathbf{0 . 9 9 L}$, respectively. Thereafter, this vortex induces further smaller ones trapped in the clear regions due to the rotating porous vanes, which act as a semi-buffer that forces the fluid particles to swirl and then flow back over the conducting surface through the porous layer covering it. Consequently, the boundary layer in the vicinity of the conducting surface becomes thinner, which leads to reactivating the heat exchange surface continually.

As the fluid flows downstream, however, the secondary flows are alleviated gradually along the main flow path until they almost vanish close to the exit of each pipe. This is attributed to the viscous dissipation and the negligibly small vorticity generation close to the fully-developed region, as inferred by Soong and Yan [33]. To justify this phenomenon, it is better to look closely at the dimensionless form of the axial vorticity transport equation presented earlier by Soong and Yan [33] but modified to take into account the foam presence as:

$$
\begin{aligned}
\frac{1}{\phi^{2}}\left[\left(U \frac{\partial \Psi}{\partial X}+V \frac{\partial \Psi}{\partial Y}+W \frac{\partial \Psi}{\partial Z}\right)+\Psi \frac{\partial U}{\partial X}\right. & \\
\left.+\left(\frac{\partial U}{\partial Y} \frac{\partial W}{\partial X}-\frac{\partial U}{\partial Z} \frac{\partial V}{\partial X}\right)\right]= & \frac{1}{\phi \operatorname{Re}}\left(\frac{\partial^{2} \Psi}{\partial X^{2}}+\frac{\partial^{2} \Psi}{\partial Y^{2}}+\frac{\partial^{2} \Psi}{\partial Z^{2}}\right)-\gamma\left(\frac{1}{D a \operatorname{Re}}+\frac{F|\mathbf{V}|}{\sqrt{D a}}\right) \Psi \\
& +\frac{2 R o}{\phi} \frac{\partial U}{\partial X}+\frac{R a_{\Omega}}{\operatorname{PrRe}^{2}}\left(Y \frac{\partial \theta_{f}}{\partial Z}-Z \frac{\partial \theta_{f}}{\partial Y}\right)
\end{aligned}
$$

Where $\Psi$ is the axial vorticity and is defined as:

$$
\Psi=\frac{\partial W}{\partial Y}-\frac{\partial V}{\partial Z}
$$


On the right side of Eq.(16), the second term refers to the vorticity dissipation due to porous resistance, while the last two ones stand for the vorticity generation terms by Coriolis influence and centrifugal buoyancy, respectively. As the axial velocity gradient is steep at the entrance regions, which in turn contributes effectively in the vorticity generation due to Coriolis effects; the secondary flows are very strong there. More downstream, however, velocity gradients start to decrease as an outcome of flow development, and hence, the decreasing Coriolis generation term cannot make up the dissipation occurred in vorticity due to the foam and viscous resistance. Thus, secondary vortices are alleviated gradually until they almost fade away at the fully developed region, where the axial gradients of velocity components become zero.

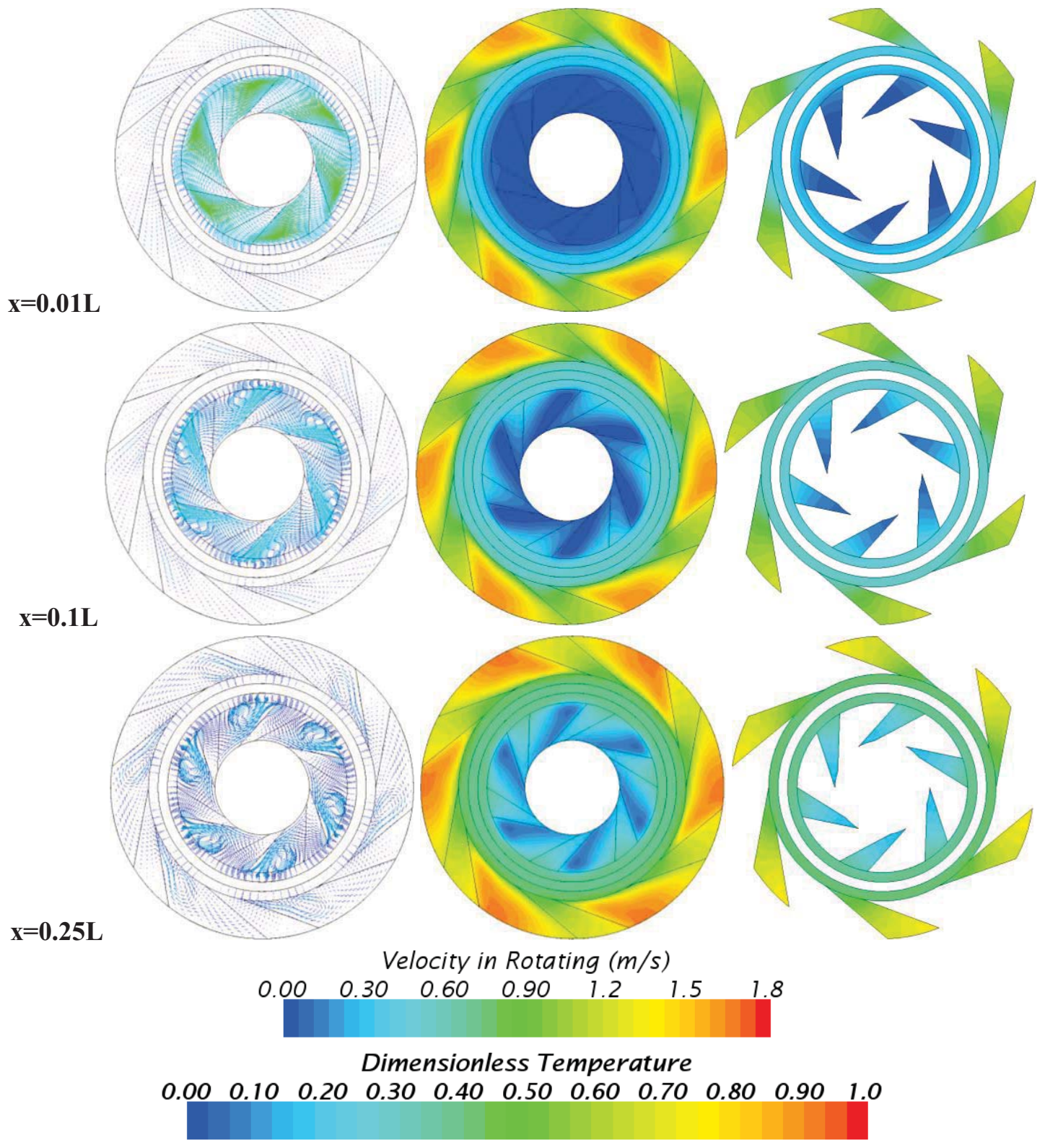

Figure 6- Development of transverse velocity vectors in rotating frame (left) and dimensionless temperature contours of fluid (middle) and solid-phase (right) at $S=2, \operatorname{Re}^{*}=1$, and $\Omega=500 \mathrm{rpm}$ 


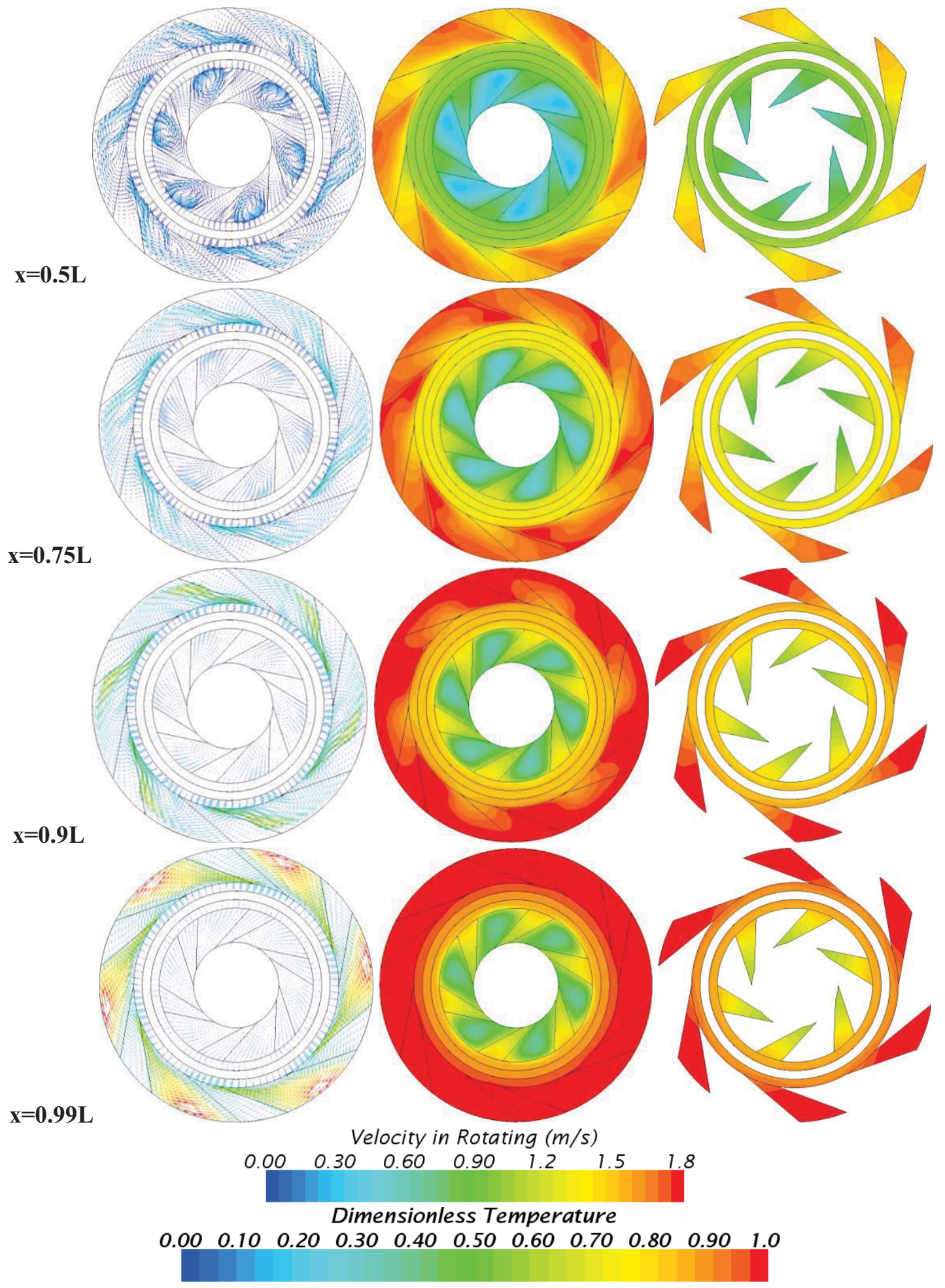

Figure 6- Continued

\subsection{Combined effects of operating conditions}

\subsubsection{Influence of rotation strength}

To explain the effects of rotation level on the flow and heat transfer patterns, the development of transverse velocity vectors are plotted in rotating frame along with the fluid temperature contours at $\boldsymbol{S}=\mathbf{2}, \boldsymbol{\phi}=\mathbf{0 . 9}, \boldsymbol{k}_{\boldsymbol{s}}=\boldsymbol{k}_{\boldsymbol{w}}, \boldsymbol{R} \boldsymbol{e}^{*}=\mathbf{1}$ for various rotation levels, as illustrated in Figure 7. In general, the stronger 
the rotational speed is applied, the better the heat exchange is achieved. Moreover, it is observed that secondary flows in the inner part are enhanced with increasing the rotation rate and sustained for a further downstream as a result of the increase in vorticity generation by Coriolis influence. The secondary vortices in the outer pipe, in contrast, look slightly weaker when rotation is enhanced. This is justified by looking closely at Eq.(16), where the higher temperature differences found in the case of lower rotational speeds result in a stronger vorticity generation due to centrifugal buoyancy and vice versa, which makes up or neutralises the dissipation along the flow path in the outer pipe.

Generally, the enhancement in heat transfer improves the effectiveness attained as shown in Figure 8. As in most heat exchangers, however, such improvement has a price, which is the increase in the pressure drop, and hence, the pumping power required. This is attributed to the augmentation in flow resistance due to the enhancement in the swirls generated across the clear and porous regions. In regard to the overall system performance achieved, it is slightly improved at the lower rotation levels, but then starts to deteriorate. This outcome is due to the fact that pressure drop increases marginally at the lower rotational speeds, while thereafter its growth becomes considerable causing to augment the pumping power significantly. Overall, the negligibly small pumping power required compared to the amount of heat exchanged makes the overall performance of such heat exchangers incomparable, i.e. $\boldsymbol{O S} \boldsymbol{P}=\boldsymbol{O}\left(1 \mathbf{1}^{2}\right)$.

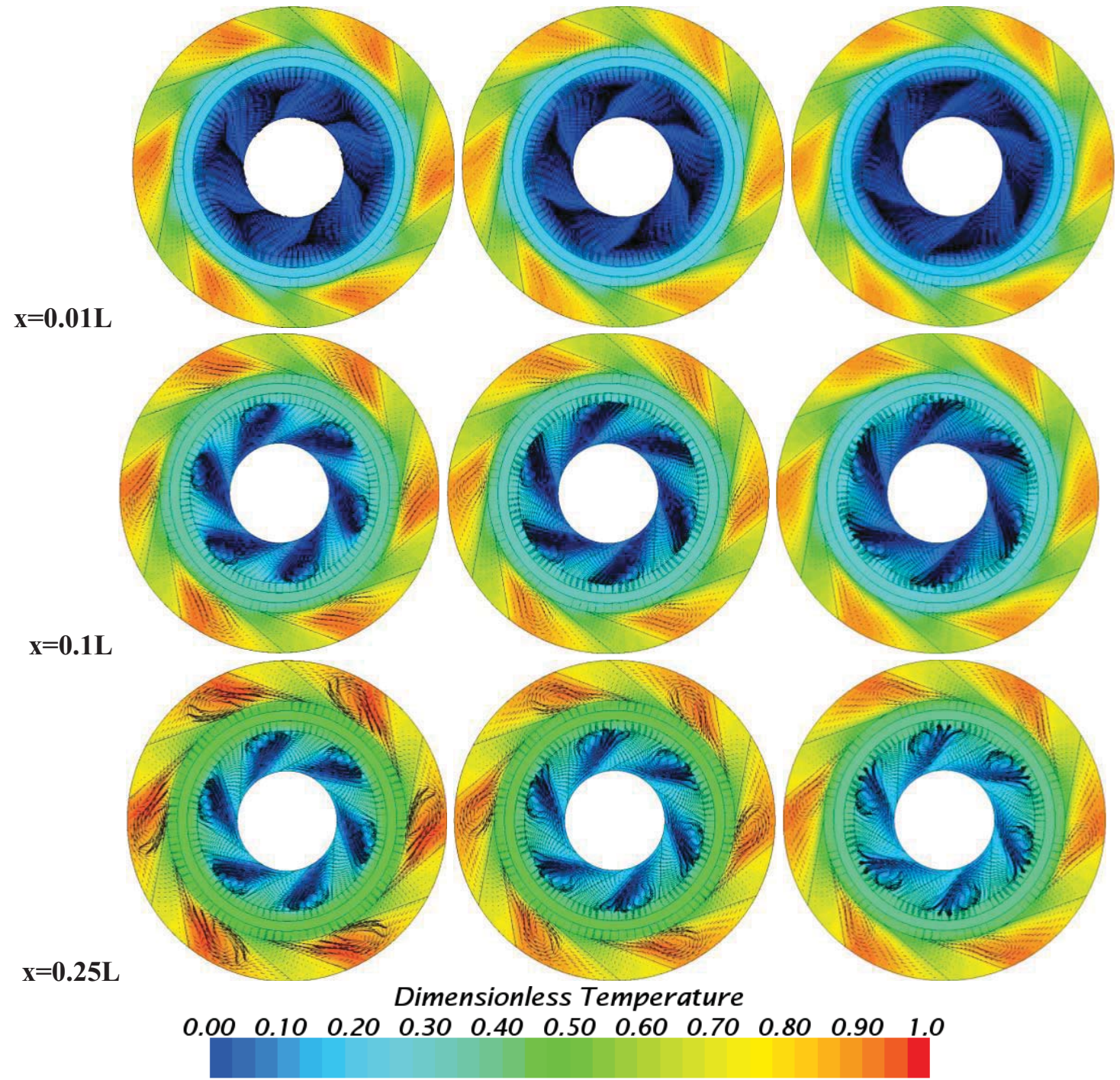

Figure 7- Velocity vectors in rotating frame and dimensionless fluid-phase temperature at $S=2$ and $\operatorname{Re}^{*}=\mathbf{1}$ for rotational speed: 125rpm (left), 250rpm (middle), and 500rpm (right) 

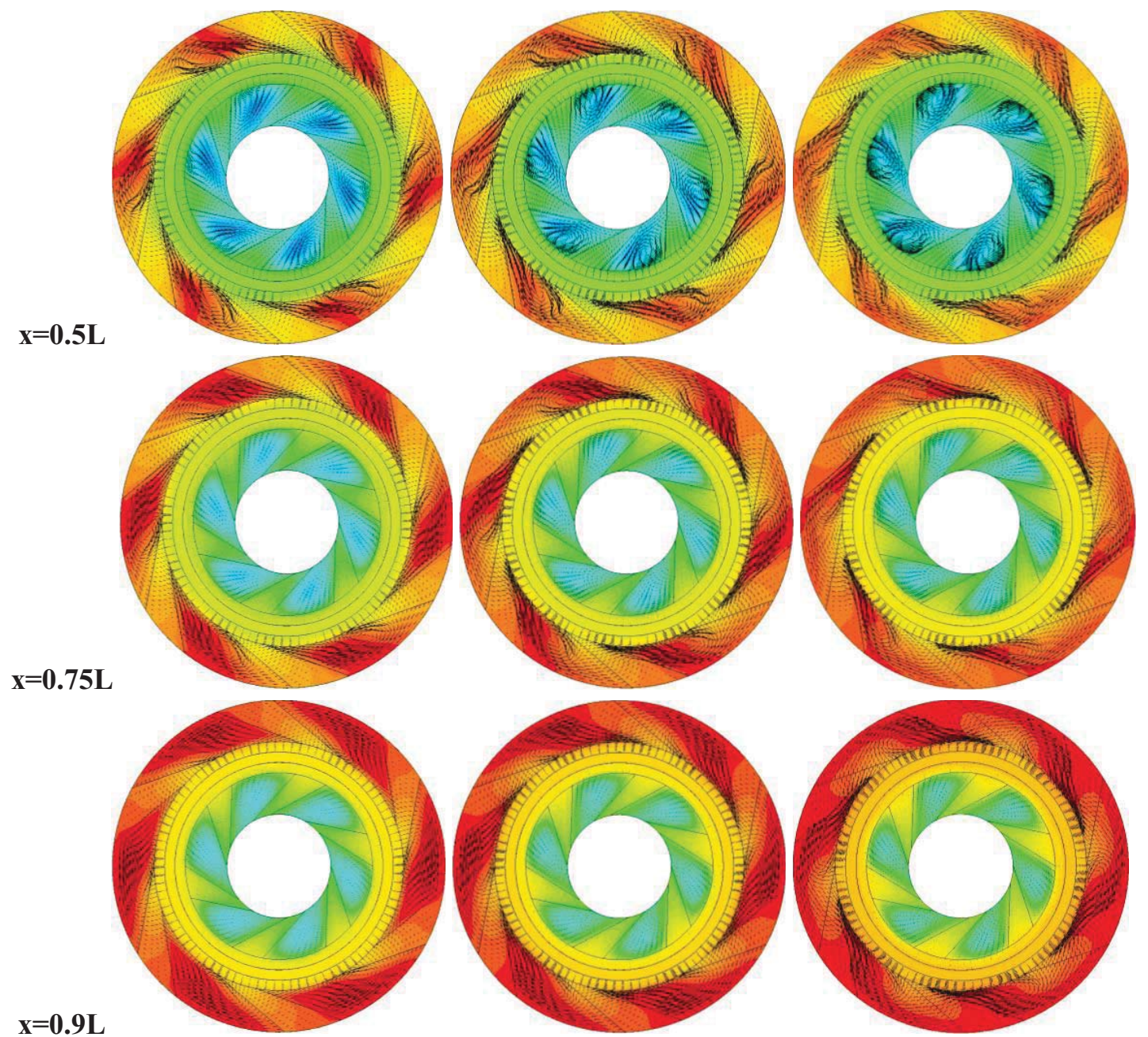

$x=0.99 L$
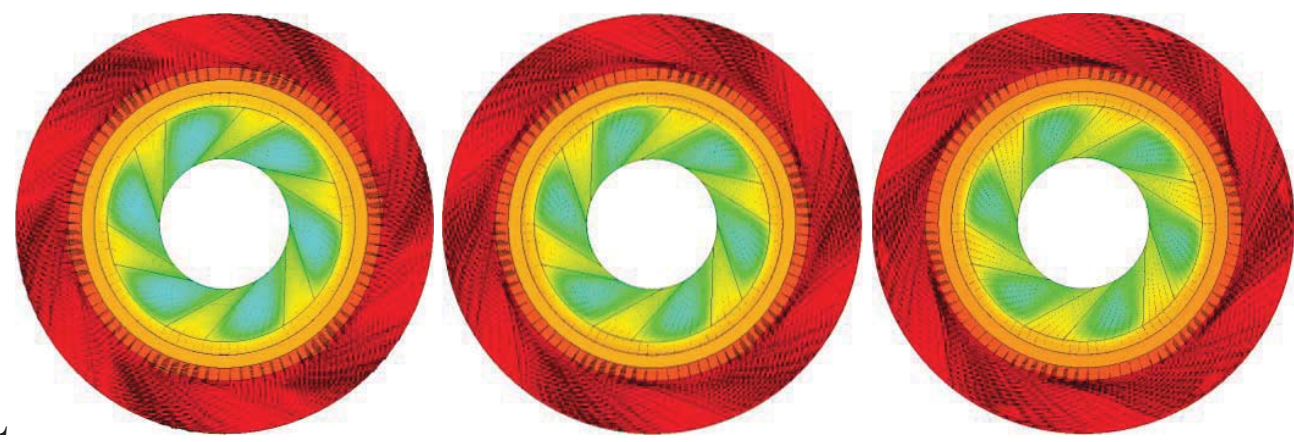

Dimensionless Temperature

$\begin{array}{lllllllllll}0.00 & 0.10 & 0.20 & 0.30 & 0.40 & 0.50 & 0.60 & 0.70 & 0.80 & 0.90 & 1.0\end{array}$

Figure 7- Continued 


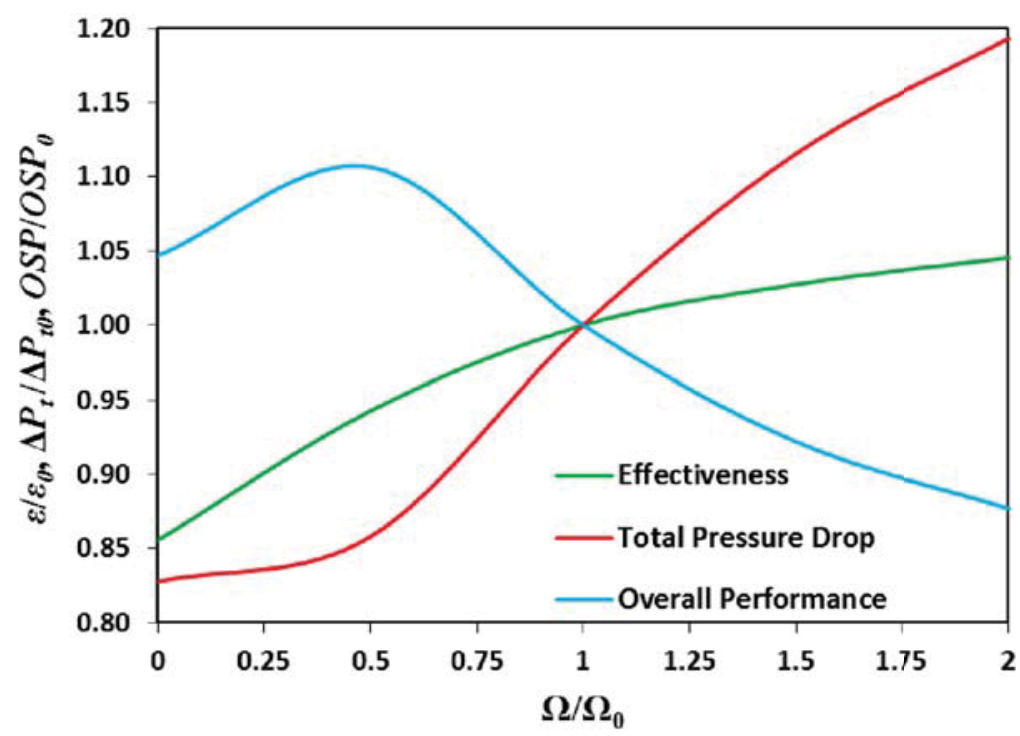

Figure 8- Influence of rotation rate on the effectiveness, total pressure drop, and overall system performance at $\Omega_{0}$ $=250 \mathrm{rpm}, \varepsilon_{0}=0.615, \Delta P_{t_{0}}=38.24 P a$, and $\mathrm{OSP}_{0}=353.56$

\subsubsection{Influence of fluid flow strength}

As explained earlier, the Reynolds number value is kept constant at the inlet to the outer part. So, the effect of changing the inner flow rate, or in other words the value of $\boldsymbol{R} \boldsymbol{e}^{*}$, is what being discussed herein. Three values of outer-to-inner Reynolds number ratio, i.e. $\boldsymbol{R} \boldsymbol{e}^{*}=\mathbf{1}, \mathbf{2}$, and $\mathbf{4}$, are compared by plotting the axial development of transverse velocity vectors and fluid temperature contours as shown in Figure 9. In general, the flow stream with the smaller heat capacity rate $\boldsymbol{C}_{\boldsymbol{m} i n}$ experiences a higher temperature change, and consequently, it will be the first to reach the maximum temperature bounds allowed, at which heat exchange comes to a halt (Kays and London [34]). Thus, the higher the $\boldsymbol{R} \boldsymbol{e}^{*}$ value, which means the lower $\boldsymbol{C}_{\boldsymbol{c}} / \boldsymbol{C}_{\boldsymbol{h}}$ ratio, the higher the exit fluid temperature from the inner pipe. In contrast, increasing the $\boldsymbol{R} \boldsymbol{e}^{*}$ value results in augmenting fluid temperature at the exit of the outer pipe. Moreover, as the inner flow velocity decreases with increasing the $\boldsymbol{R}^{*}$ value, the strength and sustainability of inner vortices is reduced as a result of the reduction in Coriolis influence. By contrast, the secondary flows become stronger and more sustainable in the outer pipe while increasing the $\boldsymbol{R e}^{*}$ value. The reason for this is that vorticity generation by centrifugal buoyancy becomes more influential due to the relatively high temperature gradients found there.

Overall, as the inner heat capacity rates decreases, not only the heat transfer effectiveness is significantly improved as illustrated in Figure 10, but a considerable saving in pressure drop is attained as well, as shown in Figure 11. However, these two gains are not reflected positively on the overall performance obtained. Instead, it is observed that the overall performance deteriorates due to the reduction in the amount of heat absorbed by the inner stream. 

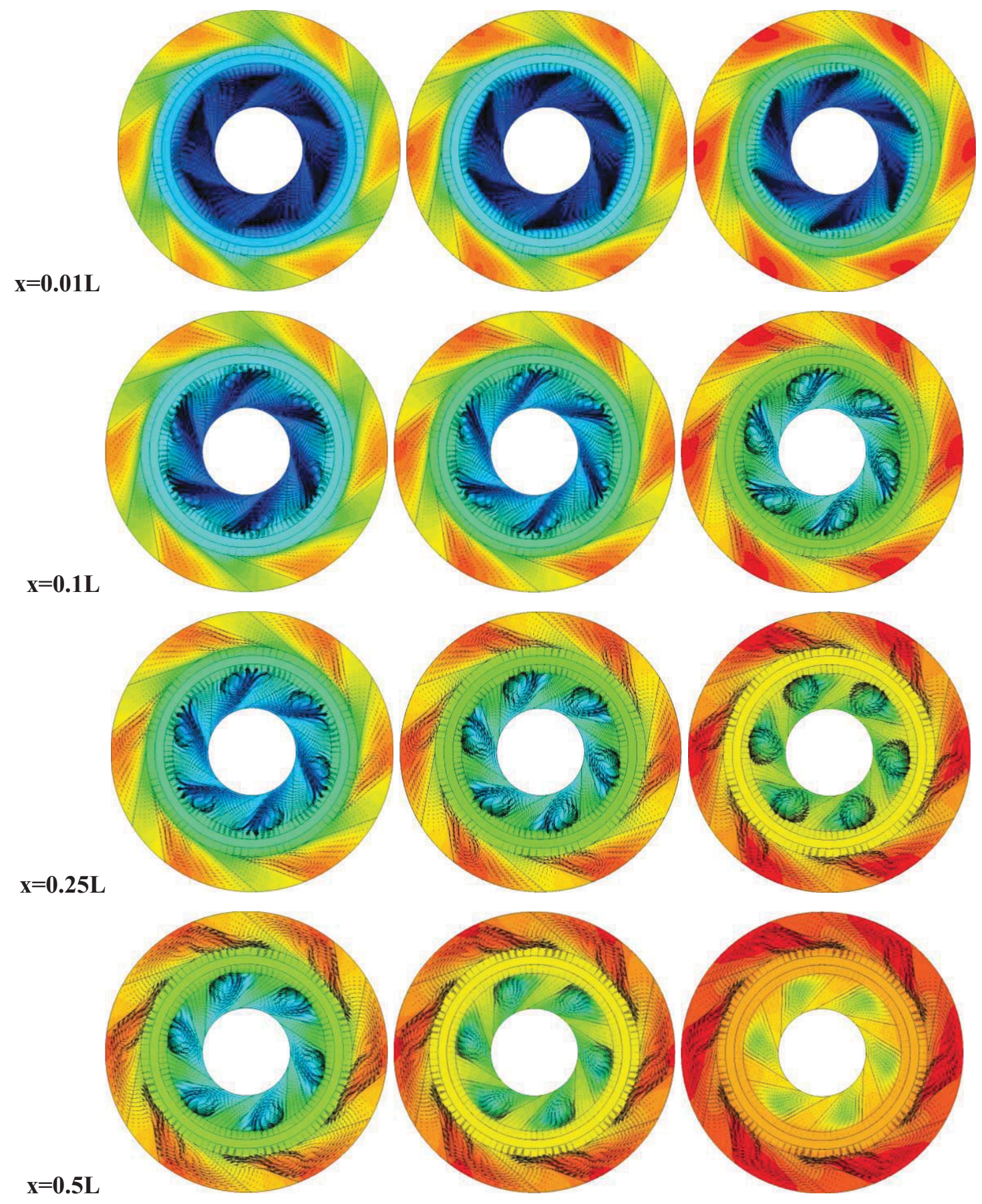

Dimensionless Temperature

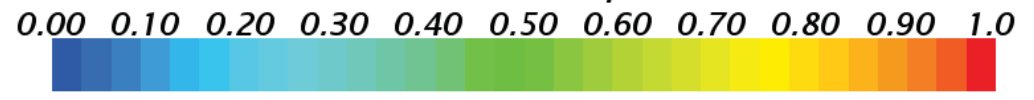

Figure 9- Velocity vectors in rotating frame and dimensionless fluid-phase temperature at $S=2$ and $\Omega=500 \mathrm{rpm}$ for Renolds number ratio: $\operatorname{Re}^{*}=1$ (left), 2 (middle), and 4 (right) 


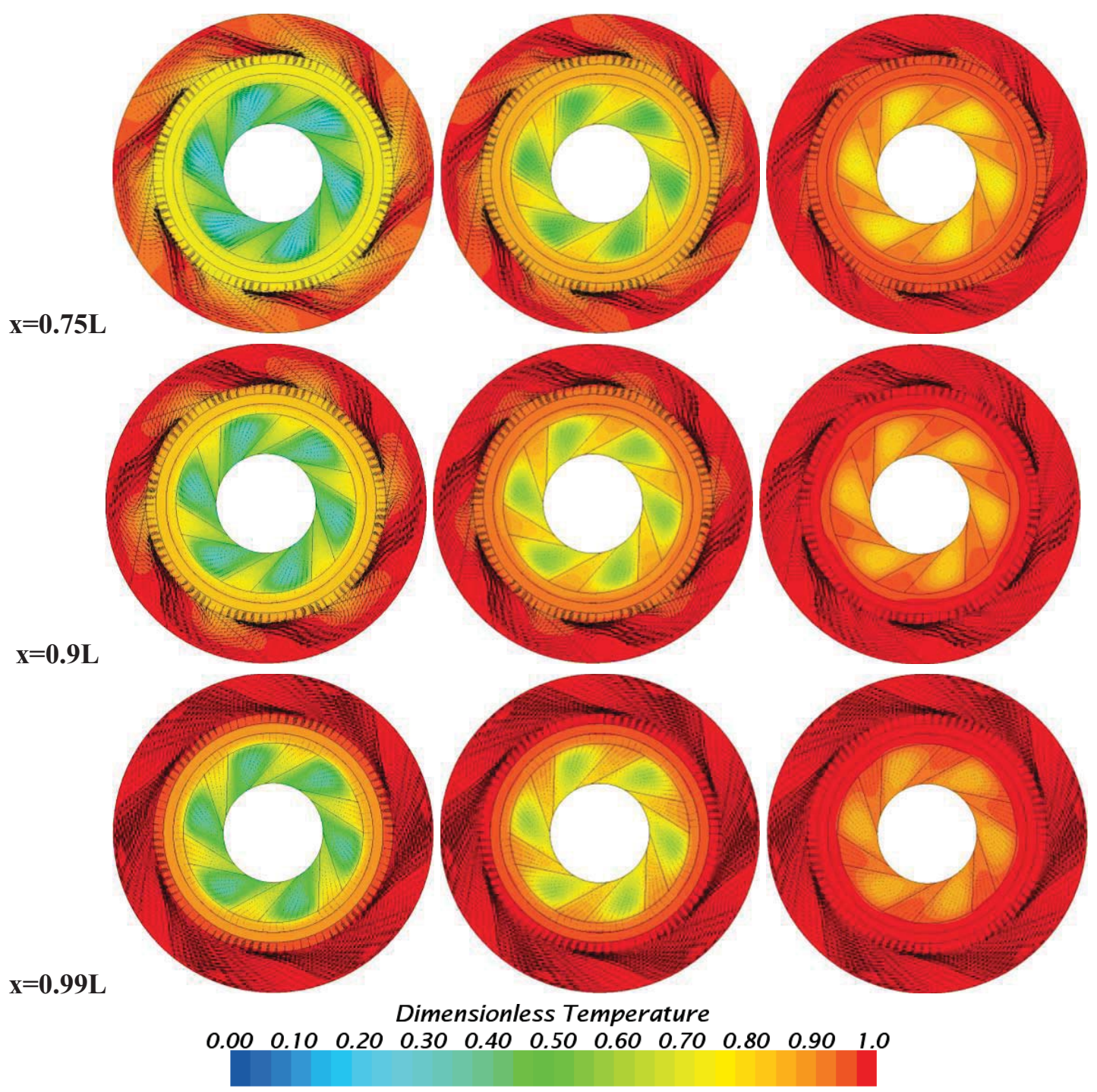

Figure 9- Continued

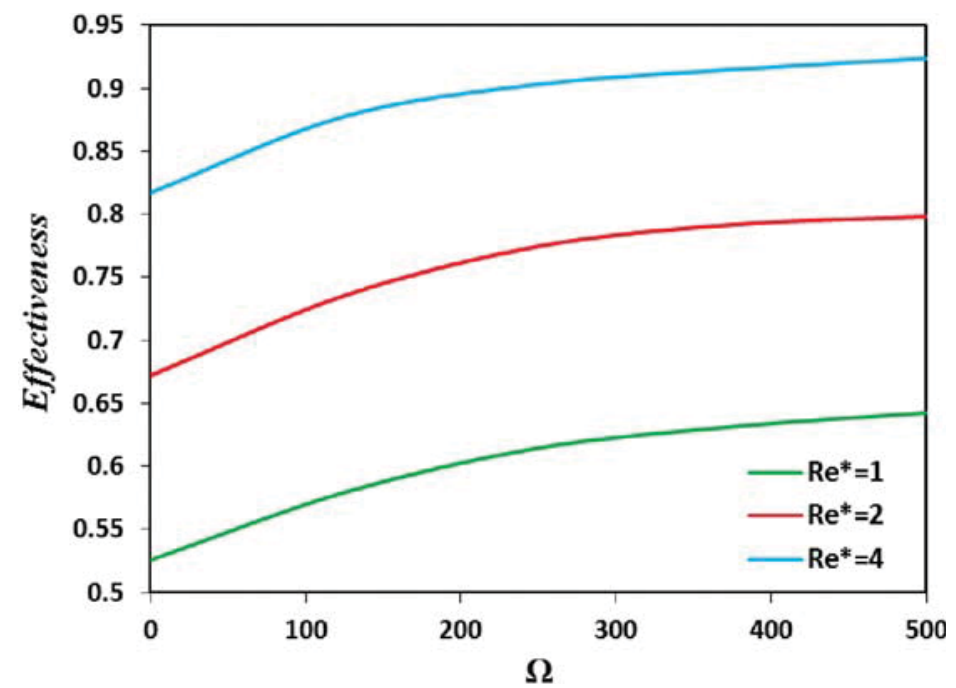

Figure 10- Influence of outer-to-inner Reynolds number ratio on the effectiveness 


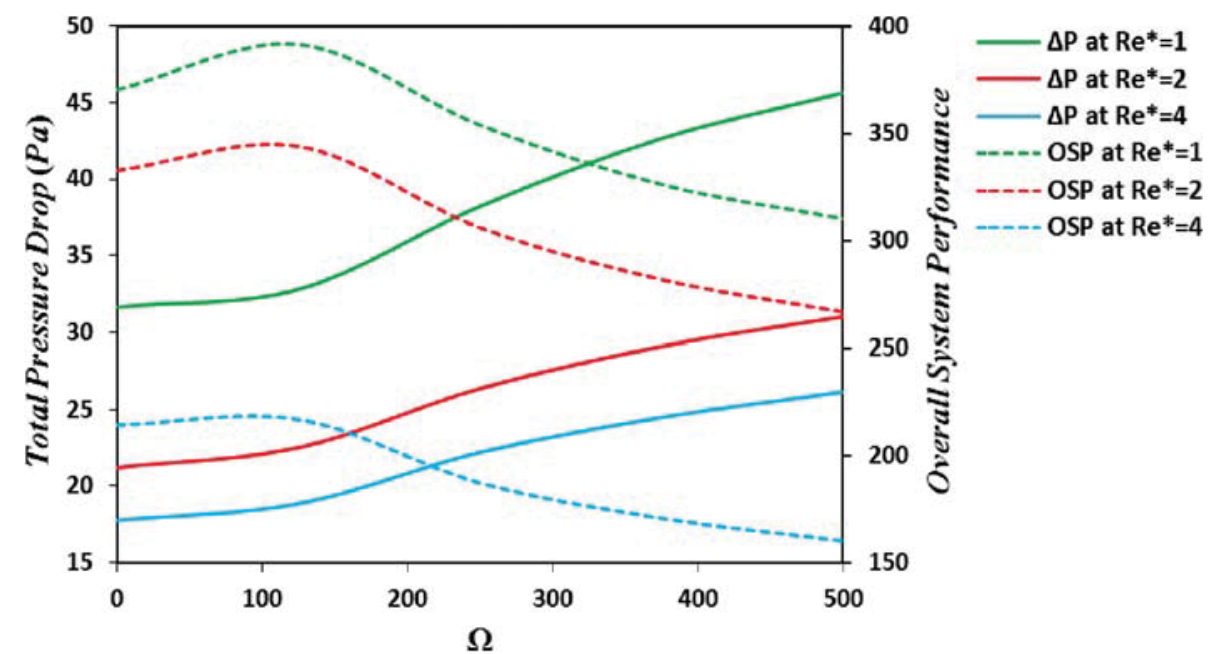

Figure 11- Impact of $\operatorname{Re}^{*}$ on the total pressure drop and overall performance achieved

\subsubsection{Influence of characteristic temperature difference}

At moderate characteristic temperature differences, i.e. $\Delta \boldsymbol{T}_{\boldsymbol{c}} \leq \mathbf{3 0}{ }^{\circ} \mathrm{C}$, fluid and solid thermophysical properties are assumed to be constant everywhere except the fluid density in the centrifugal buoyancy term, which varies according to the Boussinesq approximation. However, as the temperature difference between the heat exchanger terminals increases, it implies significant changes in the thermophysical properties. Thus, both air viscosity and thermal conductivity are considered variable with the fluid-phase temperature depending on Sutherland's law, while the density variation is estimated as a function to the temperature only using the Equation of State in order to maintain the incompressible flow conditions.

The influence of temperature difference $\left(\Delta \boldsymbol{T}_{\boldsymbol{c}}=\boldsymbol{T}_{\boldsymbol{h} \boldsymbol{1}}-\boldsymbol{T}_{\boldsymbol{c} 1}\right)$ on the effectiveness and pressure drop as well as the overall system performance, for $\boldsymbol{S}=\mathbf{2}, \boldsymbol{\Omega}=\mathbf{5 0 0} \mathbf{r p m}$, and $\boldsymbol{T}_{\boldsymbol{c l}}=\mathbf{2 0}{ }^{\circ} \mathbf{C}$, is shown in Figure 12.a,b. In general, the effectiveness is slightly improved with increasing the temperature difference. However; as both the flow velocity and dynamic viscosity increase, which results in augmenting the foams resistance to the fluid flowing across it, the pressure loss is augmented considerably. Overall, this extra cost resulting from the increase in the pumping power required looks quite marginal compared to the considerable enhancement in the amount of heat gained by the inner stream, see Figure 12.b. It is apparent that while increasing the temperature difference from 30 to $\mathbf{3 0 0}^{\circ} \mathrm{C}$, the overall performance achieved can be improved up to $\mathbf{2 0 0 - 3 0 0 \%}$ depending on the $\boldsymbol{R} \boldsymbol{e}^{*}$ value. This outcome indicates the promising prospects of utilising the proposed configuration as a recuperator in gas turbines systems.

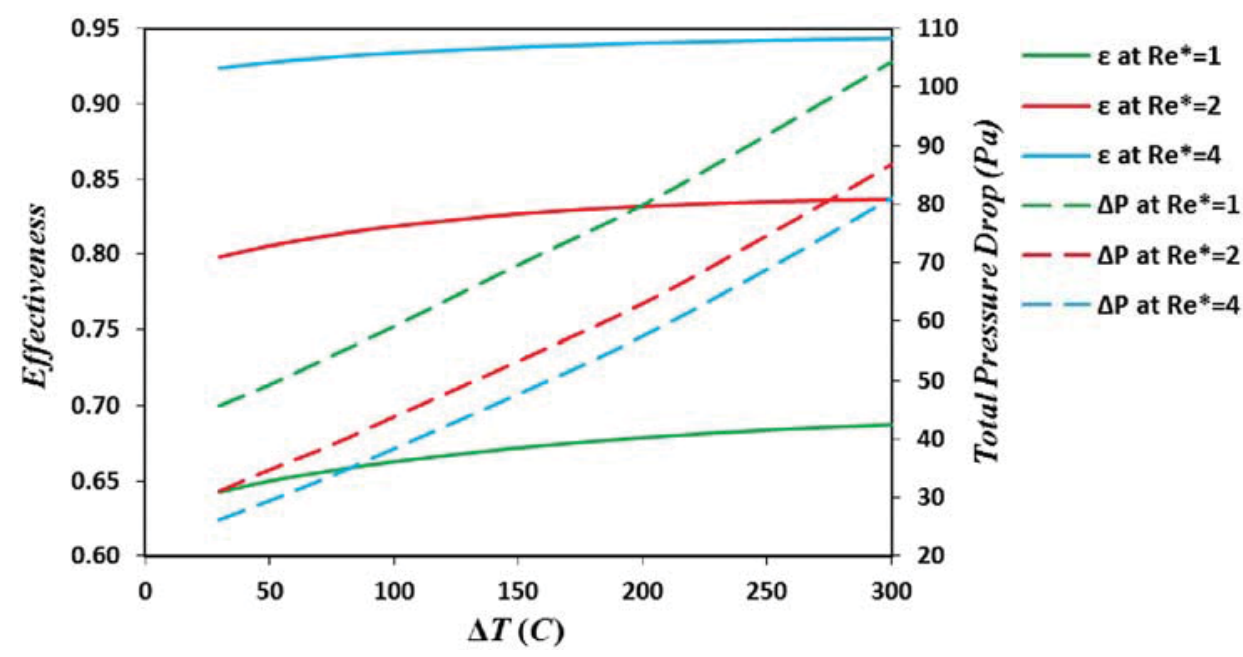

a)

Figure 12- Influence of $\Delta T_{c}$ on a) the effectiveness and pressure drop, b) the overall system performance 


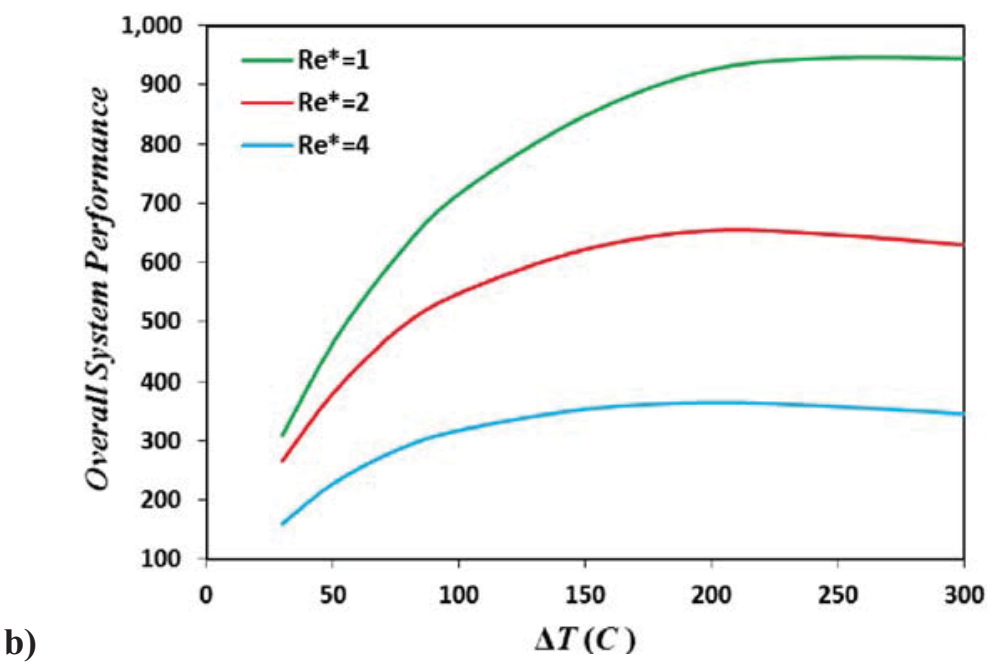

Figure 12- Continued

\subsection{Influence of hollow ratio on the fluid flow and heat transport}

To demonstrate the influence of the hollow ratio, or in other words, the clearances-to-vanes size ratio, the development of transverse velocity vectors are plotted in rotating frame along with the fluid temperature contours as illustrated in Figure 13. In general, a less heat exchange is attained when the size of clearances becomes larger. This is attributed to the reduction occurs in the mass flow across the foam region; and hence, decreasing the amount of heat transported due to the low fluid heat conductance compared to metal foams as shown in Figure 14. On the other hand, it leads to a significant saving in the pressure drop due to the reduction in the volume of the solid matrix, which acts as an obstructing or damper to the fluid flowing across it as illustrated in Figure 15. However, increasing the rotational speed $\boldsymbol{\Omega}$ does not result in the same extent of augmentation in both heat transfer and pressure drop. Depending upon the hollow ratio $S$, it is apparent that rotation becomes less influential at the lower hollow ratios. This is attributed to the fact that the foam impact dominates the rotational effects in both transferring heat and resisting the fluid flow within the pipes more occupied with metal foams. In regard to the overall performance attained, Figure 15 indicates that reducing the size of clearances improves the overall performance considerably as a result of the significant reduction occurred in the pressure drop, where it is obvious that the performance achieved in the heat exchangers semi/fully filled with metal foam, i.e. $\boldsymbol{S} \leq \mathbf{1}$, is negligibly small compared to those having larger clearances, i.e. $\boldsymbol{S} \geq \mathbf{2}$. 

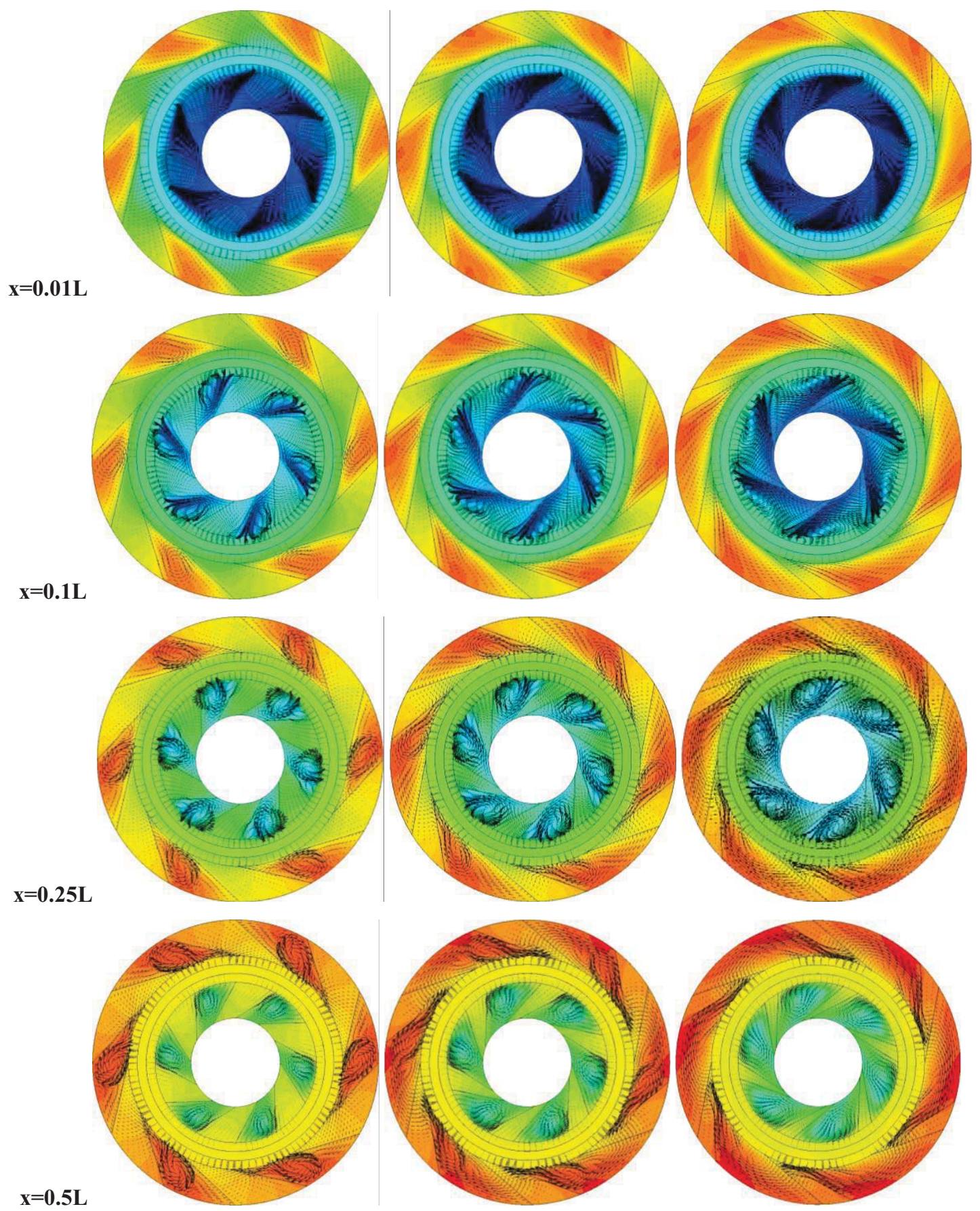

\section{Dimensionless Temperature}

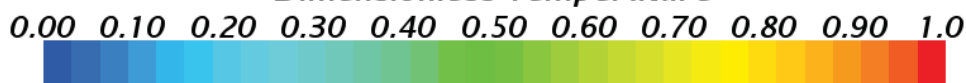

Figure 13- Velocity vectors in rotating frame and dimensionless fluid-phase temperature at $\operatorname{Re}^{*}=\mathbf{2}$ and $\Omega=500 \mathrm{rpm}$ for hollow ratio: $\mathrm{S}=1$ (left), 2 (middle), and 4 (right) 


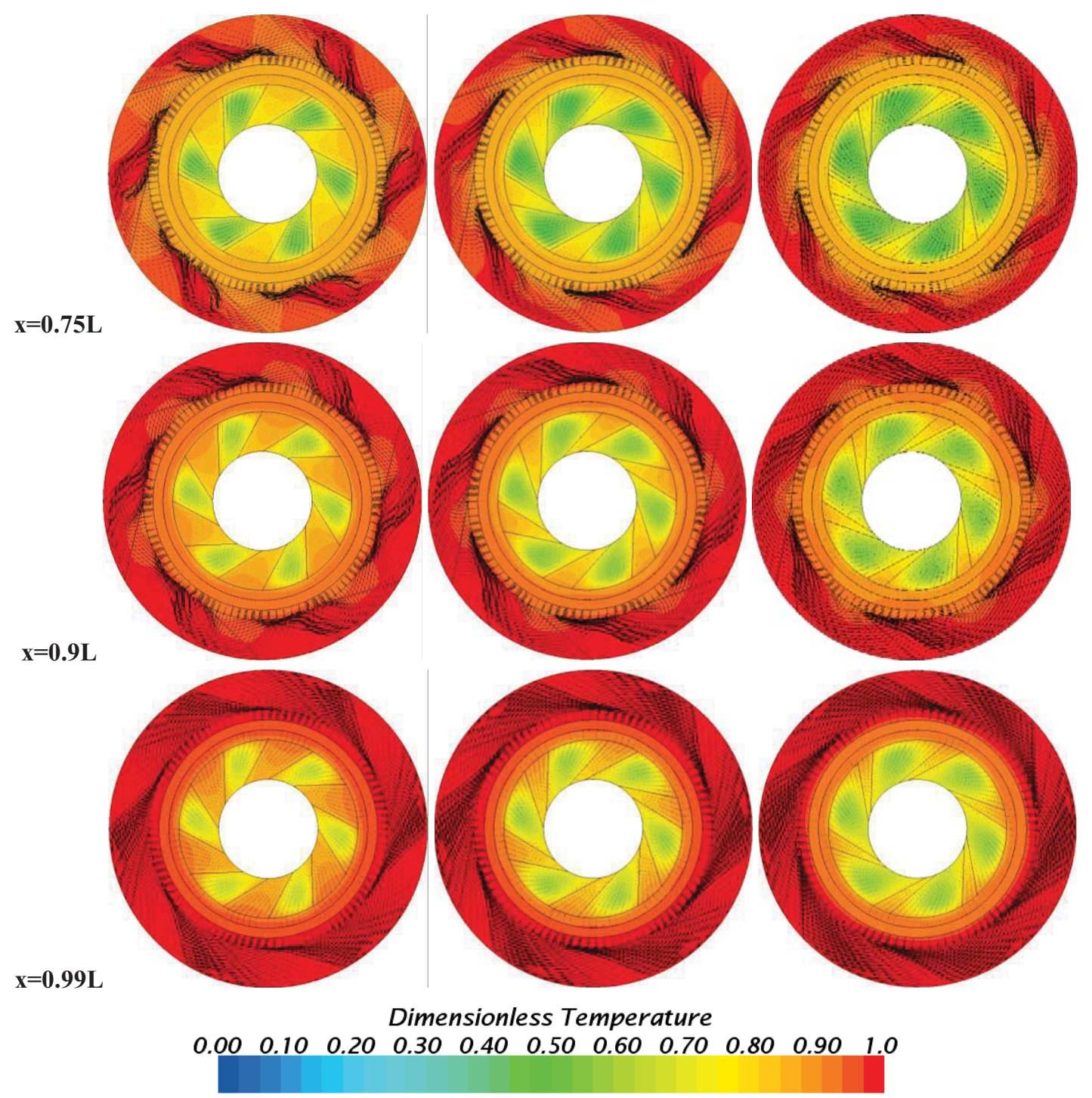

Figure 13- Continued

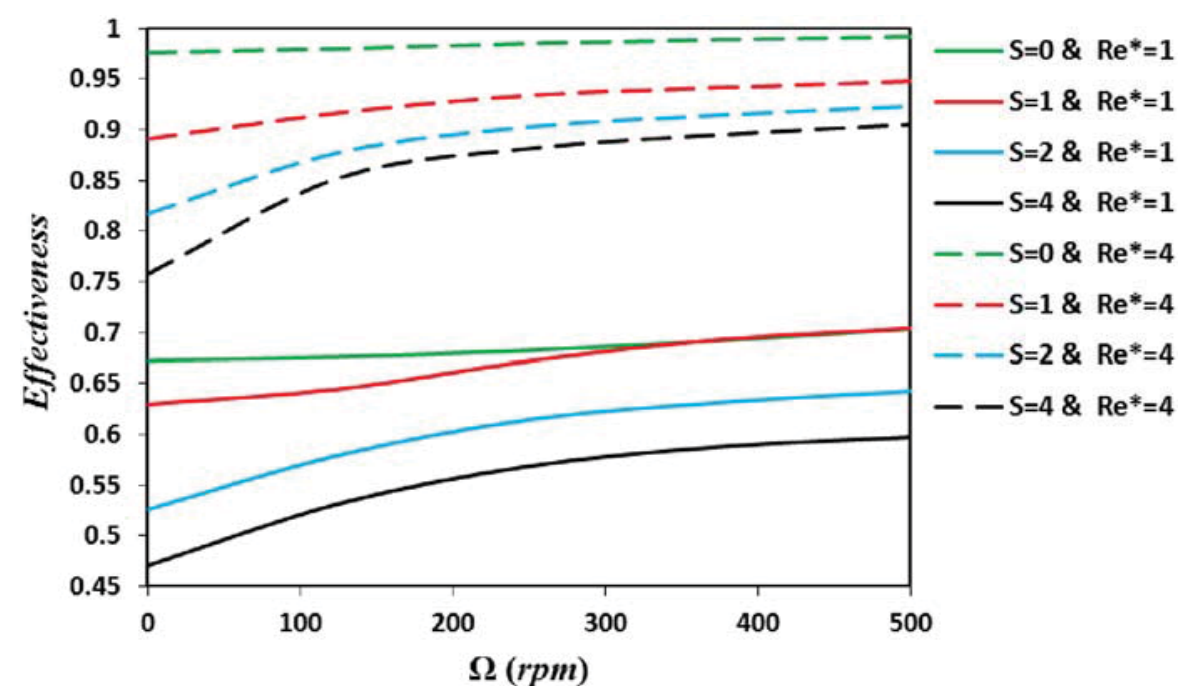

Figure 14- Hollow size influence on the effectiveness achieved 


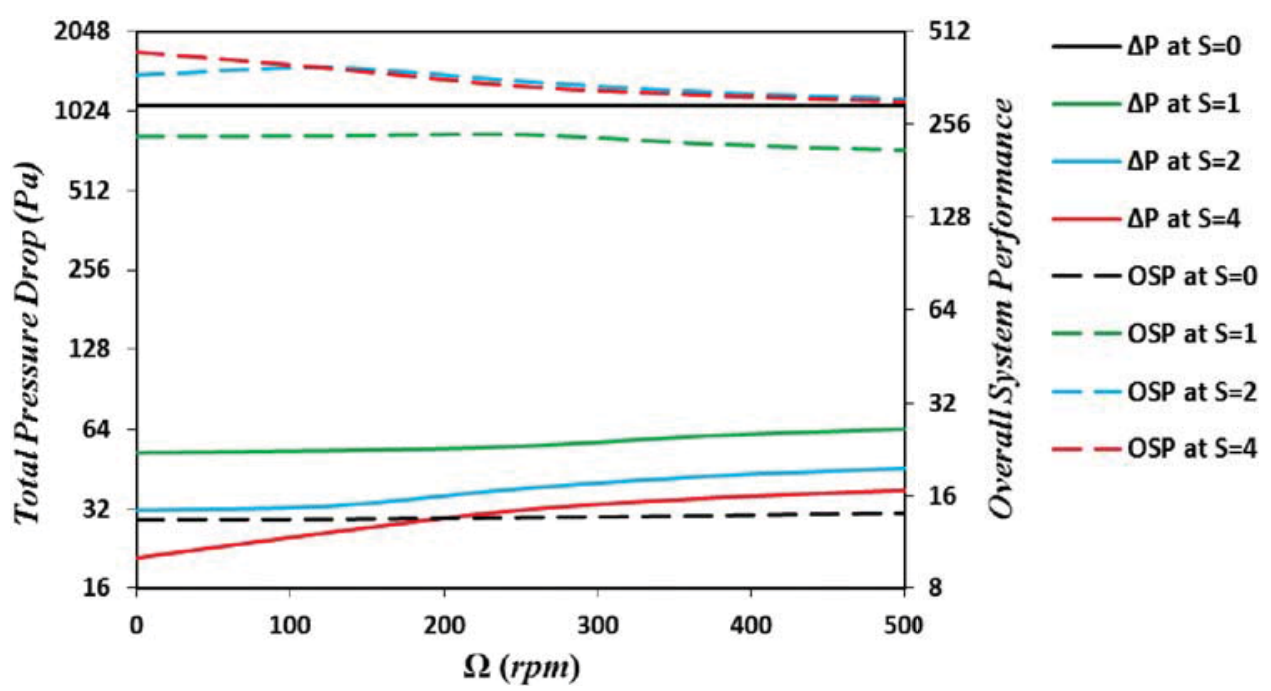

(a)

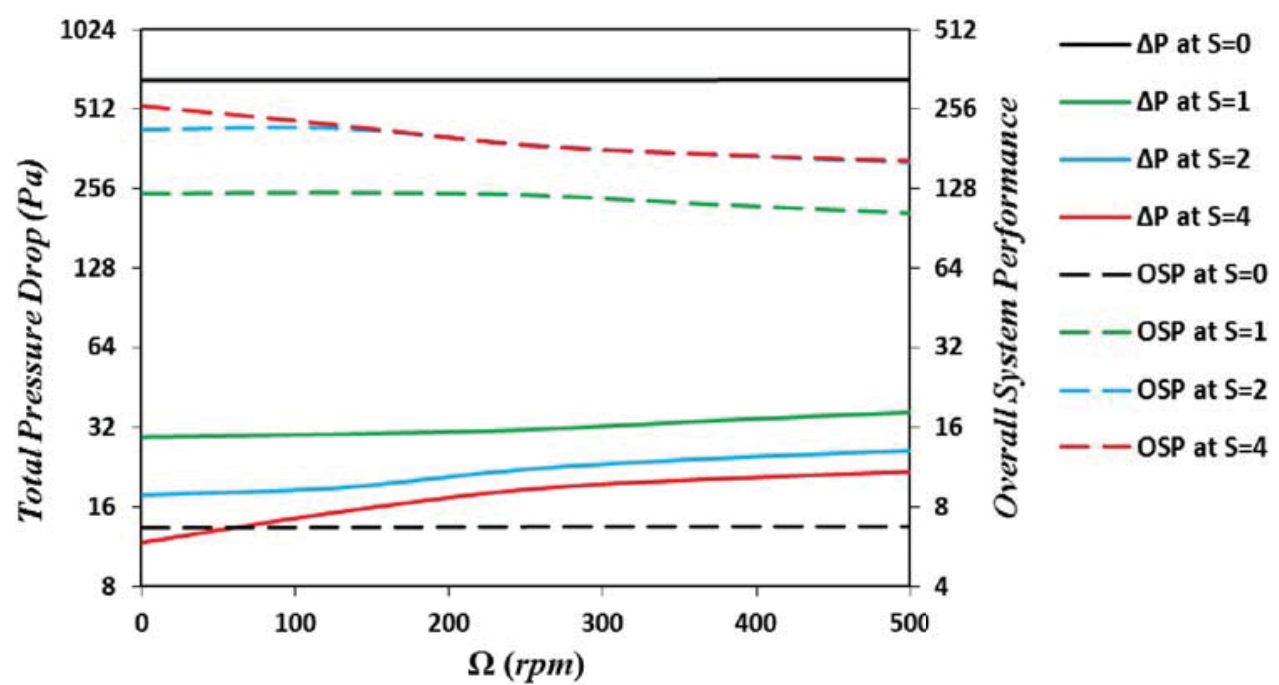

(b)

Figure 15- Effects of hollow size on both total pressure drop and the overall performance for: a) $\operatorname{Re}^{*}=1$, and b) $\operatorname{Re}^{*}=4$

\subsection{Combined influence of foam porosity and thermal conductivity}

Combined effects of foam porosity and thermal conductivity accompanying with rotation on heat transfer and the overall performance achieved are illustrated in Figure 16.a-c. In general, if the foam porosity is fixed, the amount of heat exchanged is enhanced when the conductivity ratio increases. This is owing to the increase in the foam effective conductivity, which in turn results in augmenting the overall capability of the fluid-solid medium to circulate more heat between the two streams.

As the effective foam thermal conductivity is improved with decreasing the foam porosity, it seems reasonable at first glance that heat transfer effectiveness will be enhanced as well. Apparently, this is 
true if foams with a relatively low thermal conductivity are used, e.g. stainless steel, where more heat can be exchanged, and hence, a better overall system performance is achieved. For this limited case, it is observed that porosity change becomes more influential when thinner vanes are utilised. However, this is not the fact for the case of low porosity foam formed from materials having a relatively high conductivity, i.e. aluminium. Surprisingly, it is noticed that using such sort of foams with a relatively low porosity, $\phi=\mathbf{0 . 8 9}$, causes deterioration in the effectiveness obtained compared to the corresponding foams having a higher porosity, $\phi=\mathbf{0 . 9 7}$. This unexpected outcome is attributed to the foam axial thermal conduction, which in particular becomes much stronger in the case of fully/semi filled pipes and/or the low values of inner flow rate, leading to a 'short-circuiting' across the heat exchanger. So, more heat is conducted away via the outer-pipe outlet rather than being transported to the inner-pipe across the conducting surface separating them. To overcome this drawback, the proposal presented by $\mathrm{Wu}$ et al. [35] can be utilised. So, the foams structures can be split into multi-blocks placed alternately along the heat exchanger and separated by gaps to reduce the longitudinal thermal conduction between the axially neighbouring foam elements.

In general, influence of the porosity and foam thermal conductivity on both the heat transfer and overall system performance is alleviated with decreasing either the clearances volume or the inner flow rate.

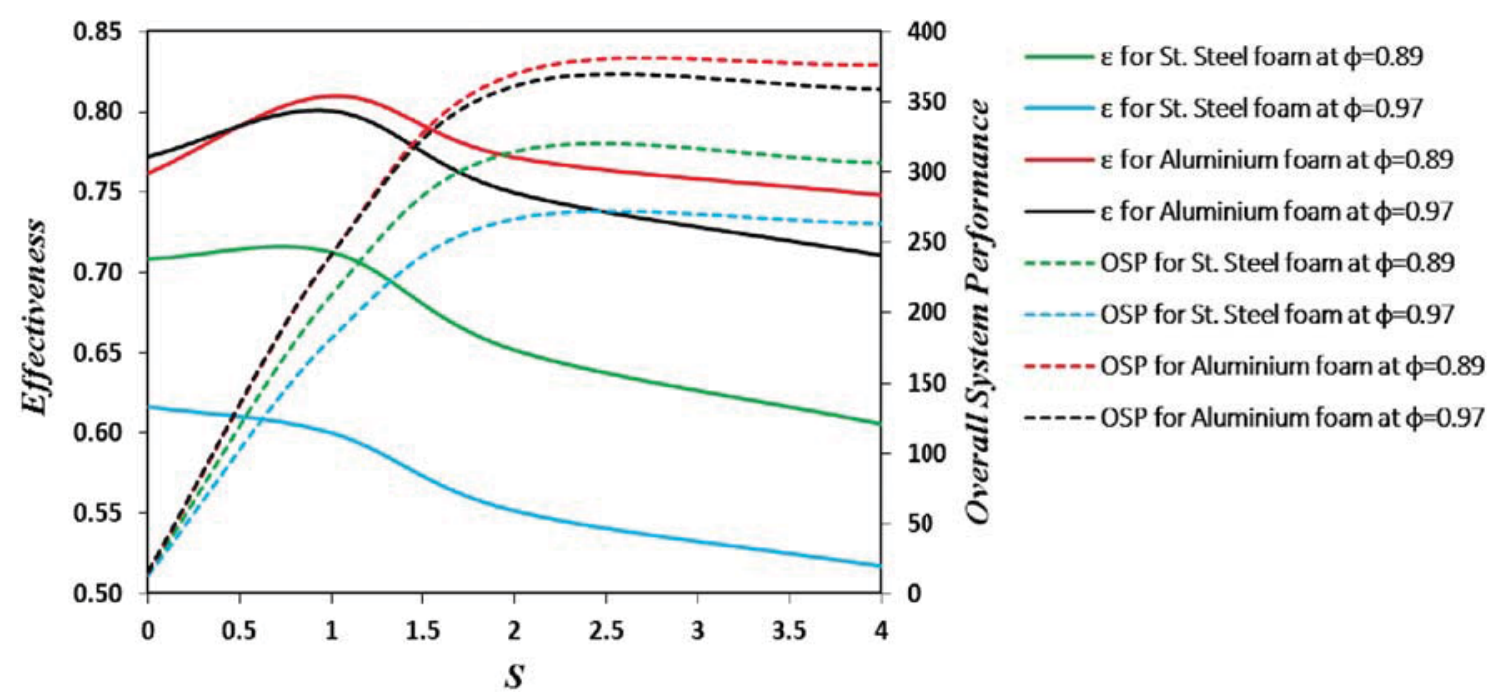

(a)

Figure 16- Effects of foam porosity and thermal conductivity on heat transfer effectiveness and overall system performance for various hollow ratios at a) $\operatorname{Re}^{*}=1$, b) $\operatorname{Re}^{*}=2$, c) $\operatorname{Re}^{*}=4$ 

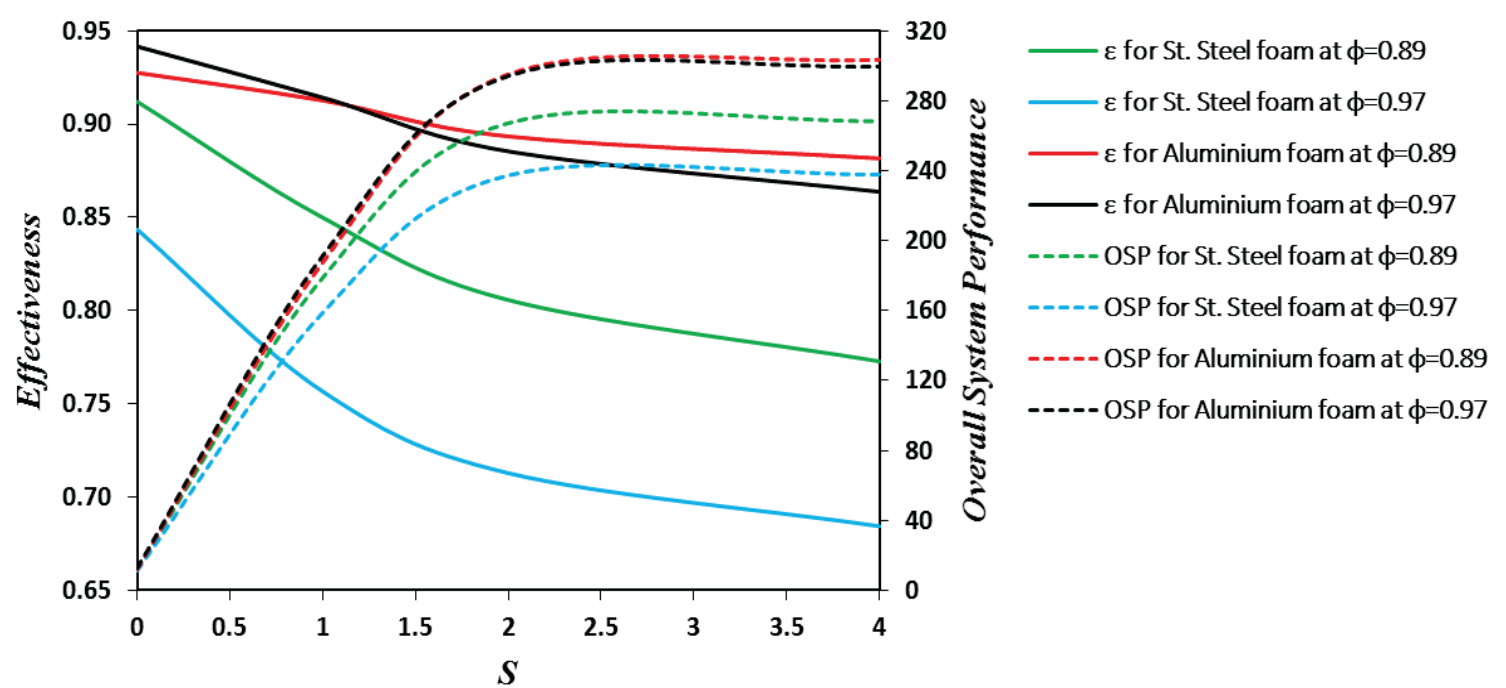

(b)
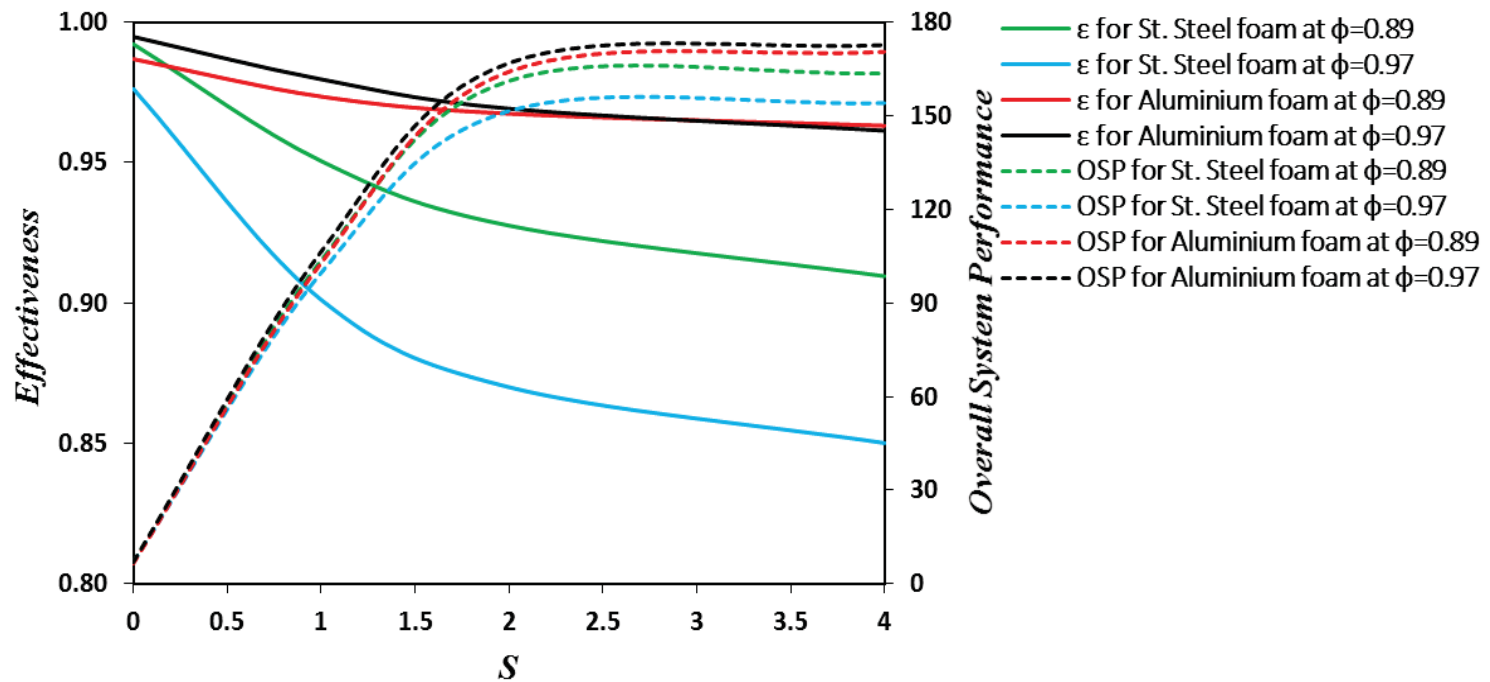

(c)

Figure 16- Continued

\section{Conclusions}

In the current study, a compound enhancement for the heat transported in a double-pipe heat exchanger is proposed through utilising both active and passive methods. The modification introduces secondary vortices in the vicinity of the conducting surface using metal foam guiding vanes. The role of these obliquely fixed vanes is to trap fluid particles while rotation and then force them to flow over the conducting surface. The other enhancement is via covering the conducting surface between the two pipes with a metal foam layer to improve the heat conductance across it.

This proposal is examined numerically by studying the three-dimensional, steady, incompressible, and laminar convective fluid flow in a counter-flow double-pipe heat exchanger partially filled with high porosity metal foam and rotating coaxially. Computations are performed for a wide range of design parameters influencing the performance achieved such as the operating conditions, the configuration of the guiding vanes utilized, and the geometrical and thermal characteristics of the metal foam utilised. 
The current proposal has "effectively" validated its potential to enhance the heat transported considerably with saving significant amount of the pumping power required compared to the corresponding heat exchangers, which are fully filled with metal foam. Also, the data obtained reveal an obvious impact of the design parameters inspected on both the heat exchanged and the pressure loss; and hence, the overall performance obtained. In general, the heat transfer can be improved considerably by manipulating the design factors, however, care must be taken to avoid conflicting uneconomical optimisation resulting from potential increases in pressure drop.

\section{Acknowledgements}

The financial support provided by the Higher Committee for Education Development in Iraq and the University of Kufa is greatly acknowledged.

\section{References}

[1] A. Alhusseny and A. Turan, "An effective engineering computational procedure to analyse and design rotary regenerators using a porous media approach," Int. J. Heat Mass Transfe, vol. 95, pp. 593-605, 2016.

[2] G. F. Hewitt, G. L. Shires, and T. R. Bott, Process Heat Transfer. USA: CRC Press, Inc., 1994.

[3] A. E. Bergles, "The Implications and Challenges of Enhanced Heat Transfer for the Chemical Process Industries," Chem. Eng. Res. Des., vol. 79, no. 4, pp. 437-444, 2001.

[4] C. Yildiz, Y. Bíçer, and D. Pehlívan, "Influence of fluid rotation on the heat transfer and pressure drop in double-pipe heat exchangers," Appl. Energy, vol. 54, no. 1, pp. 49-56, 1996.

[5] C. Yildiz, Y. Bíçer, and D. Pehlivan, "Effect of twisted strips on heat transfer and pressure drop in heat exchangers," Energy Convers. Manag., vol. 39, pp. 331-336, 1998.

[6] A. Ali, K. Vafai, and A.-R. A. Khaled, "Comparative study between parallel and counter flow configurations between air and falling film desiccant in the presence of nanoparticle suspensions," Int. J. ENERGY Res., vol. 27, pp. 725-745, 2003.

[7] A. Ali, K. Vafai, and A.-R. A. Khaled, "Analysis of heat and mass transfer between air and falling film in a cross flow configuration," Int. J. Heat Mass Transf., vol. 47, pp. 743-755, 2004.

[8] M. K. Alkam and M. A. Al-Nimr, "Improving the performance of double-pipe heat exchangers by using porous substrates," Int. J. Heat Mass Transf., vol. 42, no. 19, pp. 3609-3618, 1999.

[9] N. Targui and H. Kahalerras, "Analysis of fluid flow and heat transfer in a double pipe heat exchanger with porous structures," Energy Convers. Manag., vol. 49, no. 11, pp. 3217-3229, 2008.

[10] N. Targui and H. Kahalerras, "Analysis of a double pipe heat exchanger performance by use of porous baffles and pulsating flow," Energy Convers. Manag., vol. 76, pp. 43-54, 2013.

[11] D. A. Nield and A. Bejan, Convection in Porous Media, 4th ed. New York: Springer, 2013.

[12] D. B. Ingham and I. Pop, Eds., Transport Phenomena in Porous Media III. Oxford: Elsevier Ltd, 2005.

[13] L. Tianjian, "Ultralight porous metals: From fundamentals to applications," Acta Mech. Sin., vol. 18, no. 5, pp. 457-479, 2002.

[14] K. Boomsma, D. Poulikakos, and F. Zwick, "Metal foams as compact high performance heat exchangers," Mech. Mater., vol. 35, no. 12, pp. 1161-1176, 2003.

[15] S. Mahjoob and K. Vafai, "A synthesis of fluid and thermal transport models for metal foam heat exchangers," Int. J. Heat Mass Transf., vol. 51, no. 15-16, pp. 3701-3711, 2008.

[16] T.-M. Jeng, S.-C. Tzeng, and R. Xu, "Experimental study of heat transfer characteristics in a 180deg round turned channel with discrete aluminum-foam blocks," Int. J. Heat Mass Transf., vol. 71, pp. 133-141, 2014.

[17] A. Alhusseny and A. Turan, "Effects of centrifugal buoyancy on developing convective laminar 
flow in a square channel occupied with a high porosity fibrous medium," Int. J. Heat Mass Transf., vol. 82, pp. 335-347, 2015.

[18] A. Alhusseny, A. Turan, A. Nasser, and F. Hidri, "Hydrodynamically and thermally developing flow in a rectangular channel filled with a high porosity fiber and rotating about a parallel axis," Int. Commun. Heat Mass Transf., vol. 67, pp. 114-123, 2015.

[19] A. Alhusseny, A. Turan, and A. Nasser, "Developing convective flow in a square channel partially filled with a high porosity metal foam and rotating in a parallel-mode," Int. J. Heat Mass Transf., vol. 90, pp. 578-590, 2015.

[20] H. J. Xu, Z. G. Qu, and W. Q. Tao, "Numerical investigation on self-coupling heat transfer in a counter-flow double-pipe heat exchanger filled with metallic foams," Appl. Therm. Eng., vol. 66, no. 1-2, pp. 43-54, 2014.

[21] X. Chen, F. Tavakkoli, and K. Vafai, "Analysis and Characterization of Metal Foam-Filled Double-Pipe Heat Exchangers," Numer. Heat Transf. Part A Appl., vol. 68, no. 10, pp. 10311049, 2015.

[22] P. Vadasz, "Flow in Rotating Porous Media," in Fluid Transport in Porous Media, P. Du Plessis, Ed. Southampton: Computational Mechanics Publications, 1997, pp. 161-214.

[23] A. Alhusseny and A. Turan, "A numerical study of double-diffusive flow in a long rotating porous channel," Heat Mass Transf., vol. 51, pp. 497-505, 2015.

[24] S.-C. Tzeng, C.-Y. Soong, and S.-C. Wong, "Heat transfer in rotating channel with open cell porous aluminium foam," Int. Commun. Heat Mass Transf., vol. 31, no. 2, pp. 261-272, 2004.

[25] V. V. Calmidi, "Transport phenomena in high porosity metal foams," University of Colorado, 1998.

[26] K. Boomsma and D. Poulikakos, "On the effective thermal conductivity of a three- dimensionally structured Fluid-saturated metal foam," Int. J. Heat Mass Transf., vol. 44, pp. 827-836, 2001.

[27] K. Boomsma and D. Poulikakos, "Corrigendum for the paper: K. Boomsma, D. Poulikakos, 'On the effective thermal conductivity of a three-dimensionally structured fluid-saturated metal foam' [International Journal of Heat and Mass Transfer, 44 (2001) 827-836]," Int. J. Heat Mass Transf., vol. 54, no. 1-3, pp. 746-748, 2011.

[28] M. L. Hunt and C. L. Tien, "Effects of thermal dispersion on forced convection in fibrous media," Int. J. Heat Mass Transf., vol. 31, no. 2, pp. 301-309, Feb. 1988.

[29] V. V. Calmidi and R. L. Mahajan, "Forced Convection in High Porosity Metal Foams," J. Heat Transfer, vol. 122, no. 3, pp. 557-565, 2000.

[30] W. Lu, C. Y. Zhao, and S. A. Tassou, "Thermal analysis on metal-foam filled heat exchangers. Part I: Metal-foam filled pipes," Int. J. Heat Mass Transf., vol. 49, no. 15-16, pp. 2751-2761, 2006.

[31] H. J. Xu, Z. G. Qu, T. J. Lu, Y. L. He, and W. Q. Tao, "Thermal Modeling of Forced Convection in a Parallel-Plate Channel Partially Filled With Metallic Foams," J. Heat Transfer, vol. 133, pp. 092603 1-092603 9, 2011.

[32] J. A. Ochoa-Tapia and S. Whitaker, "Heat transfer at the boundary between a porous medium and a homogeneous fluid," Int. J. Heat Mass Transf., vol. 40, no. 11, pp. 2691-2707, 1997.

[33] C. Y. Soong and W. M. Yan, "Development of secondary flow and convective heat transfer in isothermal/iso-flux rectangular ducts rotating about a parallel axis," Int. J. Heat Mass Transf., vol. 42, pp. 497-510, 1999.

[34] W. M. Kays and A. L. London, Compact Heat Exchangers, 3rd ed. New York: McGraw-Hill, 1984.

[35] W. Wu, J. H. Du, Y. R. Lin, L. C. Chow, and W. Notardonato, "Design and Experiment of Compact and Effective Carbon Foam Recuperative Heat Exchangers," J. Thermophys. heat Transf., vol. 23, pp. 339-345, 2009. 